\title{
Asymmetric synthesis of corsifuran A by an enantioselective reduction
}

Harry Adams, Nathan J. Gilmore, Simon Jones, ${ }^{*}$ Mark P. Muldowney, ${ }^{\dagger}$ Stephan H. von Reuß $\aleph^{\S}$ and Ramesh Vemula

Department of Chemistry, University of Sheffield, Dainton Building, Brook Hill, Sheffield. UK. S6 1XD.

${ }_{\dagger}^{\dagger}$ Shasun Pharma Solutions Ltd., Dudley, Northumberland. UK. NE23 7QG.

${ }^{\S}$ Institute Für Organische Chemie, Universität Hamburg. Martin-Luther-King-Platz 6,D-29146 Hamburg, Germany.

*Corresponding author: simon.jones@sheffield.ac.uk

\section{Contents}

\section{Supplementary Information}

Preparation of 2-bromo-5-methoxyphenylacetic acid 4

Preparation of 1-(4-methoxyphenyl)-2-(2-bromo-4-

methoxyphenyl)ethanone 3

Preparation of racemic 1-(2-bromo-5-methoxy-phenyl)-2-(3-

methoxy-phenyl)-ethanol 2

Preparation of $2 S$-(2-bromo-5-methoxy-phenyl)-1-(4-methoxy-

phenyl)-ethanol 2

Preparation of 2R-(2-bromo-5-methoxy-phenyl)-1-(4-methoxy-

phenyl)-ethanol 2

Preparation of 3, 4-dimethoxydeoxybenzoin 8

Preparation of 1-(4-methoxy-phenyl)-2-(3-methoxy-phenyl)-ethanol

$\mathrm{C}-\mathrm{O}$ bond formation

Copies of ${ }^{1} \mathrm{H}$ NMR data

$\begin{array}{ll}\text { Copies of chromatograms } & \text { S20 }\end{array}$

$\mathrm{X}$-Ray Structures 


\section{Preparation of 2-bromo-5-methoxyphenylacetic acid $4^{1}$}

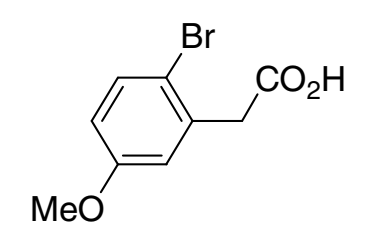

Bromine (4.31 g, $1.39 \mathrm{~mL}, 27 \mathrm{mmol}$ ) was added at $0{ }^{\circ} \mathrm{C}$ to a solution of 3 methoxyphenylacetic acid $(4.50 \mathrm{~g}, 27 \mathrm{mmol})$ in dichloromethane $(25 \mathrm{~mL})$ and stirred at room temperature for $24 \mathrm{hrs}$. The dark red solution was discoloured with sodium thiosulfate solution $(1 \mathrm{M}, 15 \mathrm{~mL})$, washed with water $(20 \mathrm{~mL})$ and separated. The aqueous layer was extracted into dichloromethane $(2 \times 25 \mathrm{~mL})$ and the combined organic layers dried over $\mathrm{MgSO}_{4}$, filtered and evaporated to dryness to give compound 4 (6.62 g, 100\%) as a white solid. mp $114{ }^{\circ} \mathrm{C}$ (lit. ${ }^{1} 114-115{ }^{\circ} \mathrm{C}$ ); IR 2974 (brd), 1690, 1596, 1571, $1473 \mathrm{~cm}^{-1} ;{ }^{1} \mathrm{H}$ NMR $\left(250 \mathrm{MHz} ; \mathrm{CDCl}_{3}\right) \delta_{\mathrm{H}} 7.75-8.30(1 \mathrm{H}$, brd), $7.45(1 \mathrm{H}, \mathrm{d}, J$ 9.0), $6.83(1 \mathrm{H}, \mathrm{d}, J 3.0), 6.70(1 \mathrm{H}, \mathrm{dd}, J 9.0,3.0), 3.55(3 \mathrm{H}, \mathrm{s})$, $3.50(2 \mathrm{H}, \mathrm{s}) ;{ }^{13} \mathrm{C}$ NMR (63 MHz; $\left.\mathrm{CDCl}_{3}\right) \delta_{\mathrm{C}} 177.1,158.9,134.3,133.4,117.3,115.1$, 114.8, 55.5, 41.6; HRMS calcd for $\mathrm{C}_{9} \mathrm{H}_{9} \mathrm{O}_{3}{ }^{79} \mathrm{Br}\left[\mathrm{M}^{+}\right]$243.9735, found 243.9739; MS (EI) 246 (25), 244 (25), 201 (20), 199 (20), 166 (18), 165 (100), 121 (20), 109 (15).

\section{Preparation of 1-(4-methoxyphenyl)-2-(2-bromo-4-methoxyphenyl)ethanone $3^{2}$}

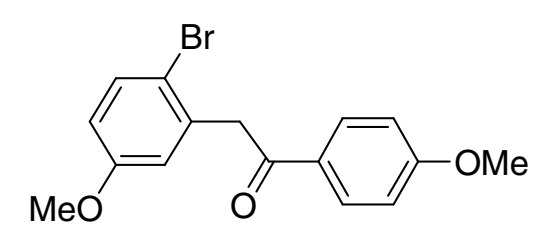

2-Bromo-5-methoxyphenyl acetic acid $4(312 \mathrm{mg}, 1.3 \mathrm{mmol})$ and thionyl chloride (155 mg, $1.3 \mathrm{mmol}$ ) were combined in a round-bottomed flask and refluxed for 30 mins. The reaction was then evaporated under a reduced pressure to dryness, redissolved in dichloromethane $(10 \mathrm{~mL})$ and cooled to $-5{ }^{\circ} \mathrm{C}$. Anisole $(140 \mathrm{mg}, 1.3$ mmol) was added followed by the addition of powdered aluminium trichloride (173 $\mathrm{mg}, 1.3 \mathrm{mmol}$ ) portion-wise. The reaction was stirred for $30 \mathrm{mins}$ and then poured into a small beaker of ice, the organic layer separated and the aqueous layer extracted with dichloromethane $(2 \times 10 \mathrm{~mL})$. The combined organic fractions were washed with $1 \mathrm{M} \mathrm{HCl}(30 \mathrm{~mL})$ and dried over $\mathrm{MgSO}_{4}$. After purification by chromatography on silica gel, eluting with $20 \%$ EtOAc / $80 \%$ petroleum ether $40-60$ ), compound 3 was obtained as an off white solid (0.380 g; 89\%); mp $113-114{ }^{\circ} \mathrm{C}$ (lit. $\left.{ }^{2} 114{ }^{\circ} \mathrm{C}\right)$; IR 1672, 1596, 1574, $1459 \mathrm{~cm}^{-1} ;{ }^{1} \mathrm{H}$ NMR $\left(250 \mathrm{MHz} ; \mathrm{CDCl}_{3}\right) \delta_{\mathrm{H}} 8.08-8.00(2 \mathrm{H}, \mathrm{m})$, 
$7.48(1 \mathrm{H}, \mathrm{d}, J 8.8), 6.99-6.93(2 \mathrm{H}, \mathrm{m}) 6.78(1 \mathrm{H}, \mathrm{d}, J 2.8), 6.41(1 \mathrm{H}, \mathrm{dd}, J$ 8.0, 2.8), $4.38(2 \mathrm{H}, \mathrm{s}), 3.90(3 \mathrm{H}, \mathrm{s}) 3.78(3 \mathrm{H}, \mathrm{s}) ;{ }^{13} \mathrm{C} \mathrm{NMR}\left(63 \mathrm{MHz} ; \mathrm{CDCl}_{3}\right) \delta_{\mathrm{C}} 194.9,163.7$, 158.9, 136.2, 133.2, 130.7, 129.6, 117.2, 115.4, 114.5, 113.9, 55.5, 55.4, 45.6; HRMS calcd for $\mathrm{C}_{16} \mathrm{H}_{15} \mathrm{O}_{3}{ }^{79} \mathrm{Br}\left[\mathrm{M}^{+}\right]$334.0205, found 334.0207; MS (EI) 336 (1), 334 (1), 255 (32), 135 (100), 107 (6), 92 (15), 77 (24), 64 (6).

\section{Preparation of racemic 1-(2-bromo-5-methoxy-phenyl)-2-(3-methoxy-phenyl)- ethanol 2}

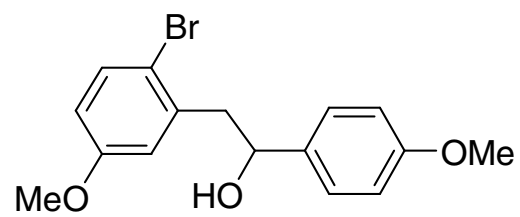

Sodium borohydride $(0.33 \mathrm{~g}, 8.9 \mathrm{mmol})$ was added to ethanol $(25 \mathrm{~mL})$ and stirred at room temperature. Ketone 3 (1.50 g, $4.4 \mathrm{mmol})$ was added drop-wise with stirring and the reaction was left for $1 \mathrm{~h}$ at room temperature. Water $(10 \mathrm{~mL})$ was added, the mixture extracted with $\mathrm{CH}_{2} \mathrm{Cl}_{2}\left(3 \times 30 \mathrm{~cm}^{3}\right)$, the organic layer washed with water $(2 \times$ $200 \mathrm{~mL}$ ) and dried over $\mathrm{MgSO}_{4}$. The solution was filtered and concentrated under reduced pressure to provide the desired product 2 (1.38 g, 92\%); Analytical data was as reported below.

\section{Preparation of 2S-(2-bromo-5-methoxy-phenyl)-1-(4-methoxy-phenyl)-ethanol 2}

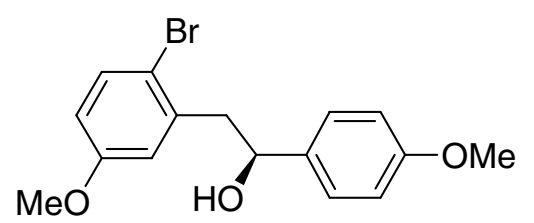

Trimethyl borate $(0.25 \mathrm{~mL}, 0.2 \mathrm{mmol})$ was added to a solution of the $(1 R, 2 S)-1$ amino-indan-2-ol (3 mg, $0.2 \mathrm{mmol})$ in THF (3 mL) and stirred at room temperature under a nitrogen atmosphere for $30 \mathrm{~min}$. $\mathrm{BH}_{3}$.DMS complex $(0.17 \mathrm{~mL}, 2.0 \mathrm{mmol})$ was added, the reaction stirred for $30 \mathrm{~min}$, then ketone $3(0.737 \mathrm{~g}, 2.19 \mathrm{mmol})$ in THF $(2 \mathrm{~mL})$ added via cannula. The reaction mixture was stirred for a further $30 \mathrm{~min}$ at room temperature then quenched with methanol $(5 \mathrm{~mL})$. Water $(10 \mathrm{~mL})$ was added and the solvent removed under reduced pressure. The mixture was extracted with $\mathrm{CH}_{2} \mathrm{Cl}_{2}(3 \times 10 \mathrm{~mL})$, the organic phase washed with $1 \mathrm{M} \mathrm{HC} 1(30 \mathrm{~mL})$, water $(30 \mathrm{~mL})$ and dried over $\mathrm{MgSO}_{4}$. Filtration, and removal of the solvent under reduced pressure 
produced the crude product $(S)-2$ in $76 \%$ ee. The alcohol was recrystallised to $99 \%$ ee from petroleum ether $(40-60) / \mathrm{CH}_{2} \mathrm{Cl}_{2} ; \mathrm{mp} 76-77^{\circ} \mathrm{C},[\alpha]_{\mathrm{D}}-11.2\left(c 1, \mathrm{CHCl}_{3}\right)$; ee 99\% [Kromasil 3-CelluCoat 8\% iso-propanol in hexane @ $1.00 \mathrm{~mL} / \mathrm{min}, 10.04$ and 15.36 mins (major)]; IR 3361, 2931, 1614, 1571, 1514, $1454 \mathrm{~cm}^{-1} ;{ }^{1} \mathrm{H}$ NMR (250 $\left.\mathrm{MHz}, \mathrm{CDCl}_{3}\right) \delta_{\mathrm{H}} 7.45(1 \mathrm{H}, \mathrm{d}, J$ 9.1), $7.36-7.29(2 \mathrm{H}, \mathrm{m}), 6.90-6.82(2 \mathrm{H}, \mathrm{m}), 6.71$ (1H, d, $J$ 2.8), 6.67 (1H, dd, J 9.1, 2.8), 4.95 (1H, dd, J 4.9, 8.5), 3.80 (3H, s), 3.72 $(3 \mathrm{H}, \mathrm{s}), 3.13(1 \mathrm{H}, \mathrm{dd}, J 4.9,13.7), 3.02(1 \mathrm{H}, \mathrm{dd}, J 8.5,13.7), 1.95\left(1 \mathrm{H}\right.$, brd s) ${ }^{13} \mathrm{C}$ NMR $\left(125 \mathrm{MHz} ; \mathrm{CDCl}_{3}\right) \delta_{\mathrm{C}} 159.1,158.7,138.6,136.0,133.3,127.0,117.4,115.2$, 114.1, 113.8, 73.1, 55.4, 55.3, 46.4; HRMS calcd for $\mathrm{C}_{16} \mathrm{H}_{17} \mathrm{O}_{3}{ }^{79} \mathrm{Br}\left[\mathrm{M}^{+}\right]$336.0361, found 336.0367; MS (EI) 338 (1), 336 (1), 202 (13), 200 (14), 137 (100), 109 (20), 94 (15), 77 (30).

\section{Preparation of 2R-(2-bromo-5-methoxy-phenyl)-1-(4-methoxy-phenyl)-ethanol 2.}

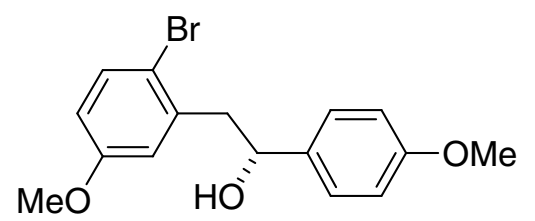

Using the above method with $(1 S, 2 R)$-catalyst with ketone 3 (2.66 g, $7.92 \mathrm{mmol})$, gave alcohol $(R)-2(2.38 \mathrm{~g}, 89 \%)$ in $78 \%$ ee. The ee was increased to $99 \%$ by repeated recrystallisation from petroleum ether $(40-60) / \mathrm{CH}_{2} \mathrm{Cl}_{2} ; \mathrm{mp} 77-78{ }^{\circ} \mathrm{C},[\alpha]_{\mathrm{D}}+15.9(c$ 1, $\mathrm{CHCl}_{3}$ ); ee 99\% [Kromasil 3-CelluCoat 8\% iso-propanol in hexane @ $1.00 \mathrm{~mL} /$ min, 9.90 (major) and 15.84 mins]; remainder of analytical data the same as for $(S)-2$.

\section{Preparation of 3, 4-dimethoxydeoxybenzoin $8^{3}$}

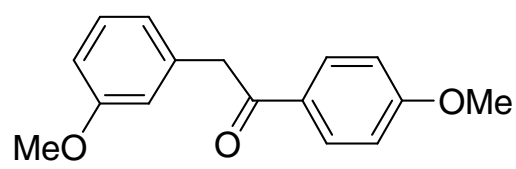

3-Methoxyphenylacetic acid (4.00 g, $24 \mathrm{mmol})$ and thionyl chloride (2.86 g, 23 mmol) were combined in a round-bottomed flask and refluxed for 30 mins. The reaction was then evaporated under a reduced pressure to dryness, re-dissolved in dichloromethane $(40 \mathrm{~mL})$ and cooled to $-5{ }^{\circ} \mathrm{C}$. Anisole $(2.60 \mathrm{~g}, 24 \mathrm{mmol})$ was added followed by the addition of powdered aluminium trichloride (3.20 g, $24 \mathrm{mmol})$ portion-wise. The reaction was stirred for 30 mins and then poured into a small beaker of ice, the organic layer separated and the aqueous layer extracted with 
dichloromethane $(2 \times 40 \mathrm{~mL})$. The combined organic fractions were washed with $1 \mathrm{M}$ $\mathrm{HCl}(40 \mathrm{~mL})$ and dried over $\mathrm{MgSO}_{4}$. After purification by chromatography on silica gel, eluting with $20 \%$ EtOAc / 80\% petroleum ether 40 - 60), compound 8 (3.90 g; $65 \%$ ) was obtained as an off white solid; mp $44-45{ }^{\circ} \mathrm{C}$ (lit. ${ }^{3} 38-40{ }^{\circ} \mathrm{C}$ ); IR 1672 , 1592, $1492 \mathrm{~cm}^{-1} ;{ }^{1} \mathrm{H}$ NMR (250 MHz; $\left.\mathrm{CDCl}_{3}\right) \delta_{\mathrm{H}} 8.02-7.95(2 \mathrm{H}, \mathrm{m}), 7.25-7.17$ $(1 \mathrm{H}, \mathrm{m}), 6.95-6.74(5 \mathrm{H}, \mathrm{m}), 4.22(2 \mathrm{H}, \mathrm{s}), 3.85(3 \mathrm{H}, \mathrm{s}) 3.80(3 \mathrm{H}, \mathrm{s}) ;{ }^{13} \mathrm{C} \mathrm{NMR}(125$ $\left.\mathrm{MHz} ; \mathrm{CDCl}_{3}\right) \delta_{\mathrm{C}} 195.9,163.3,159.6,136.3,130.8,129.4,121.6,114.9,113.6,112.1$, 55.2, 54.9, 45.1; HRMS calcd for $\mathrm{C}_{16} \mathrm{H}_{16} \mathrm{O}_{3}\left[\mathrm{M}^{+}\right], 256.1099$, found 256.1093; MS (EI) 256 (7), 136 (11), 135 (100), 121 (3), 107 (6), 92 (11), 77 18).

\section{Preparation of 1-(4-methoxy-phenyl)-2-(3-methoxy-phenyl)-ethanol 7}

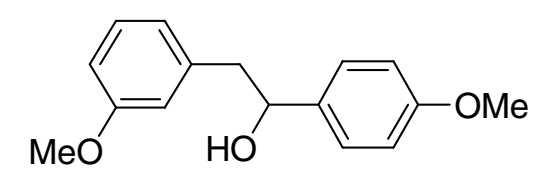

Sodium borohydride $(0.73 \mathrm{~g}, 19 \mathrm{mmol})$ was added to ethanol $(30 \mathrm{~mL})$ and stirred at room temperature. Ketone 9 ( $2.50 \mathrm{~g}, 9.7 \mathrm{mmol})$ was added drop-wise with stirring and the reaction was left for $1 \mathrm{~h}$ at room temperature. Water $(10 \mathrm{~mL})$ was added, the mixture extracted with $\mathrm{CH}_{2} \mathrm{Cl}_{2}(3 \times 30 \mathrm{~mL})$, the organic layer washed with water $(2 \times$ $200 \mathrm{~mL}$ ) and dried over $\mathrm{MgSO}_{4}$. The solution was filtered and concentrated under reduced pressure to provide the desired product 7 (2.20 g, 88\%); Found: C, 74.03; H, 7.16\%. $\mathrm{C}_{16} \mathrm{H}_{18} \mathrm{O}_{3}$ requires C, 64.39, H, 7.02; IR 3425, 2936, 1610, 1584, $1511 \mathrm{~cm}^{-1}$; ${ }^{1} \mathrm{H}$ NMR $\left(250 \mathrm{MHz} ; \mathrm{CDCl}_{3}\right) \delta_{\mathrm{H}} 7.35-7.21(3 \mathrm{H}, \mathrm{m}), 6.95-6.85(2 \mathrm{H}, \mathrm{m}), 6.81-6.72$ (4H, m), 4.85 (1H, td, J 4.7, 2.1), $3.80(3 \mathrm{H}, \mathrm{s}), 3.75$ (3H, s), $3.05-2.91$ (2H, m), 2.63 $(1 \mathrm{H}, \mathrm{d}, J 2.1) ;{ }^{13} \mathrm{C}$ NMR $\left(63 \mathrm{MHz}, \mathrm{CDCl}_{3}\right) \delta_{\mathrm{C}} 159.7,159.1,139.8,136.0,129.5$, 127.2, 121.8, 115.1, 113.8, 112.1, 74.9, 55.3, 55.2, 46.0; HRMS calcd for $\mathrm{C}_{16} \mathrm{H}_{18} \mathrm{O}_{3}$ $\left[\mathrm{M}^{+}\right]$258.1256, found 258.1252; MS (EI) 258 (4), 241 (15), 240 (23), 165 (10), 122 (100), 121 (55), 109 (55), 94 (47), 91 (52), 79 (66), 77 (95).

General procedure for preparation of Corsifuran A 1 by palladium-catalyzed intramolecular C-O bond formation<smiles>COc1ccc([C]2Cc3cc(OC)ccc3O2)cc1</smiles> 
2-Bromo-5-methoxy-phenyl-1-(4-methoxy-phenyl)-ethanol 2 (400 mg, $1.8 \mathrm{mmol}$ ) and base (1.5 equiv.), in dry toluene $(5 \mathrm{~mL})$ were added to a flame dried $50 \mathrm{~cm}^{3}$ twonecked round bottomed flask fitted with a condenser under a $\mathrm{N}_{2}$ atmosphere, containing the palladium source $(3 \mathrm{~mol} \%)$ and ligand $(3.5 \mathrm{~mol} \%)$ in toluene $(5 \mathrm{~mL})$. The reaction was refluxed for $24 \mathrm{~h}$ then cooled to room temperature, diluted with diethyl ether $(15 \mathrm{~mL})$ and filtered through Celite. The solvent was evaporated under a reduced atmosphere to give a dark yellow solution. Column chromatography on silica gel eluting with $20 \%$ EtOAc / $80 \%$ petroleum ether 40 - 60 gave natural product 1 as the first fraction, inseparable alcohols $\mathbf{2}$ and $\mathbf{7}$ as the second fraction, and inseparable ketones $\mathbf{3}$ and $\mathbf{8}$ as the third fraction. Conversion and yields were as reported in the manuscript table. Analytical data was as reported below.

\section{Preparation of $(R)$-Corsifuran A 1 by copper-catalyzed intramolecular $\mathrm{C}-\mathrm{O}$ bond formation}

(R)-2-Bromo-5-methoxy-phenyl-1-(4-methoxy-phenyl)-ethanol $2 \mathbf{2}(500 \mathrm{mg}, \quad 1.48$ mmol, $100 \%$ ee) was added to a stirred solution of sodium hydride (60\% dispersion in mineral oil; pre-washed with petroleum ether; $44 \mathrm{mg}, 1.85 \mathrm{mmol})$ in toluene $(2 \mathrm{~mL})$. The solution was heated to $40{ }^{\circ} \mathrm{C}$ for 15 mins and cooled back to room temperature. Copper $^{\mathrm{I}}$ chloride ${ }^{1}(7 \mathrm{mg}, 0.074 \mathrm{mmol})$ and ethyl acetate $(1-2$ drops $)$ in toluene $(2 \mathrm{~mL})$ were added and the reaction was heated at reflux for $24 \mathrm{~h}$. After cooling to room temperature, the solution was quenched with water $(20 \mathrm{~mL})$ and filtered through Celite with ether washings $(2 \times 10 \mathrm{~mL})$. The organic layer was separated, the aqueous layer extracted with ether $(2 \times 20 \mathrm{~mL})$ and the combined organic fractions washed sequentially with $1 \mathrm{M} \mathrm{HCl}(30 \mathrm{~mL})$, saturated $\mathrm{NaHCO}_{3}(30 \mathrm{~mL})$, saturated $\mathrm{NaCl}(30$ $\mathrm{mL})$ and water $(30 \mathrm{~mL})$ and dried over $\mathrm{NaSO}_{4}$. After flash chromatography on silica gel, eluting with $15 \%$ EtOAc / $75 \%$ petroleum ether 40-60, $(R)$-corsifuran A 1 was obtained in a $0.288 \mathrm{~g}, 76 \%$ yield, $>99 \%$ ee; (5 m octakis(2, 6-O-Me-3-O-Pe)-gammacyclodextrin capillary, hydrogen as the carrier gas at $2 \mathrm{~mL}$ per minute; Injector temp $250{ }^{\circ} \mathrm{C}$, flame ionisation detector at $250{ }^{\circ} \mathrm{C}$, isothermal at $160{ }^{\circ} \mathrm{C} ; \mathrm{mp} 61{ }^{\circ} \mathrm{C} ;[\alpha]_{\mathrm{D}}-11.5$ (c 1, $\left.\mathrm{CHCl}_{3}\right)$ [lit. $\left.{ }^{4}-11.1\left(c 0.1 \mathrm{CDCl}_{3}\right)\right]$; IR 2937, 1590, $1486 \mathrm{~cm}^{-1} ;{ }^{1} \mathrm{H}$ NMR (250 $\left.\mathrm{MHz} ; \mathrm{CDCl}_{3}\right) \delta_{\mathrm{H}} 7.33-7.22(2 \mathrm{H}, \mathrm{m}), 6.86-6.78(2 \mathrm{H}, \mathrm{m}), 6.73-6.56(4 \mathrm{H}, \mathrm{m}), 5.62$ (1H, app t, $J$ 8.8), 3.75 (3H, s), 3.70 (3H, s), 3.45 (1H, dd, $J 8.8,16.5), 3.10(1 \mathrm{H}, \mathrm{dd}, J$

\footnotetext{
${ }^{1}$ Purified reagent plus, greater than or equal to 99\%, Sigma Aldrich.
} 
8.8, 16.5); ${ }^{13} \mathrm{C}$ NMR (63 MHz; $\left.\mathrm{CDCl}_{3}\right) \delta_{\mathrm{C}} 159.4,154.2,153.7,133.9,127.7,127.3$, 113.9, 113.0, 111.1, 109.2, 84.2, 56.0, 55.3, 38.7; HRMS calcd for $\mathrm{C}_{16} \mathrm{H}_{16} \mathrm{O}_{3}\left[\mathrm{M}^{+}\right]$ 256.1099, found 256.1103; MS (EI) 256 (26), 241 (6), 213 (6), 198 (7), 181 (11), 152 (16), $115(26)$.

(S)-Corsifuran A 1; >99\% ee, (5 m octakis(2, 6-O-Me-3-O-Pe)-gamma-cyclodextrin capillary, hydrogen as the carrier gas at $2 \mathrm{~mL}$ per minute; Injector temp $250^{\circ} \mathrm{C}$, flame ionisation detector at $250{ }^{\circ} \mathrm{C}$, isothermal at $160{ }^{\circ} \mathrm{C}$; mp $61-62{ }^{\circ} \mathrm{C} ;[\alpha]_{\mathrm{D}}+15.5(c 1$, $\left.\mathrm{CHCl}_{3}\right)$.

Racemic corsifuran was prepared in an identical manner starting from racemic alcohol 2. 
${ }^{1}$ H NMR Spectrum of compound 4

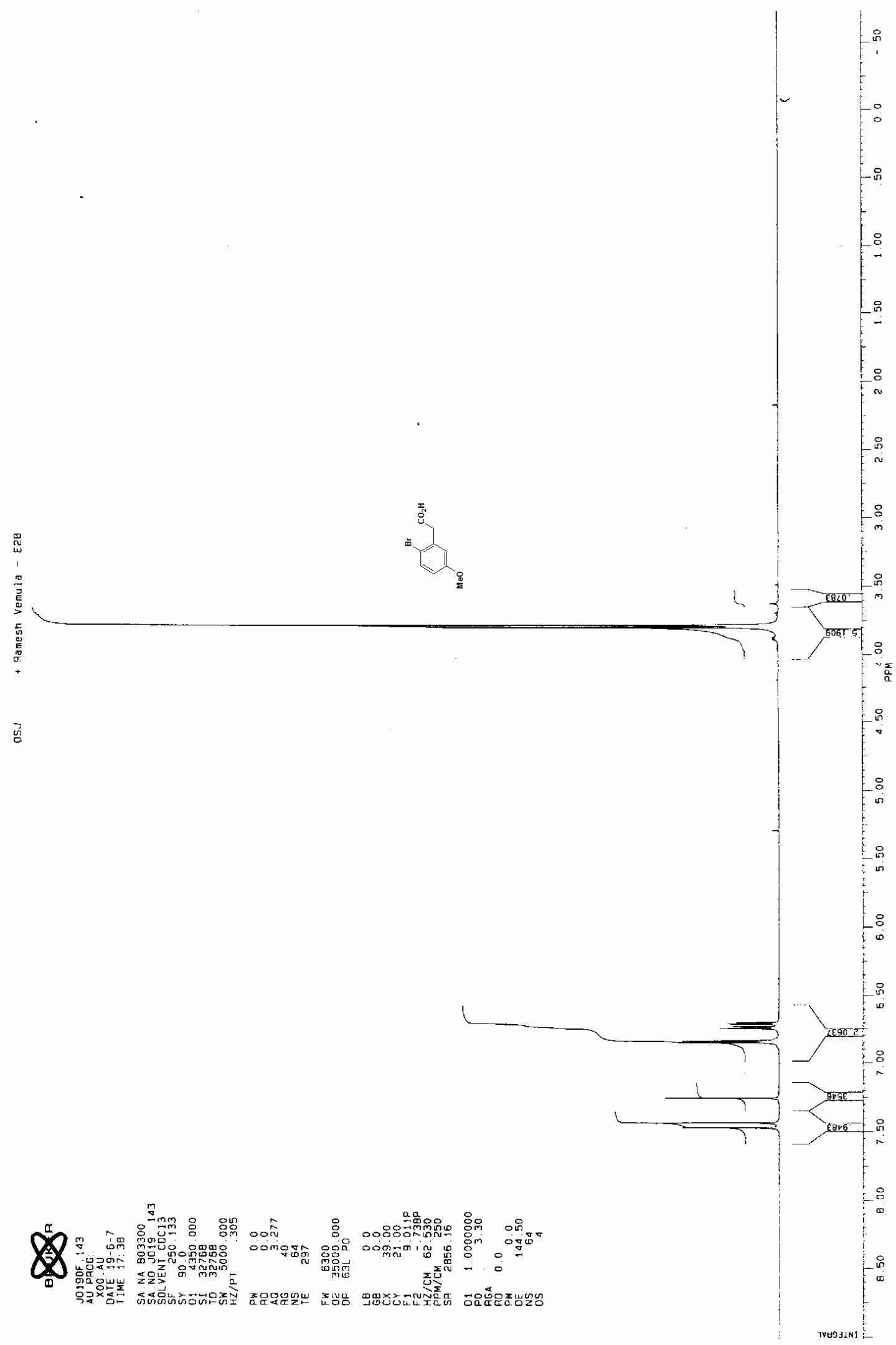


${ }^{1}$ H NMR Spectrum of compound 3

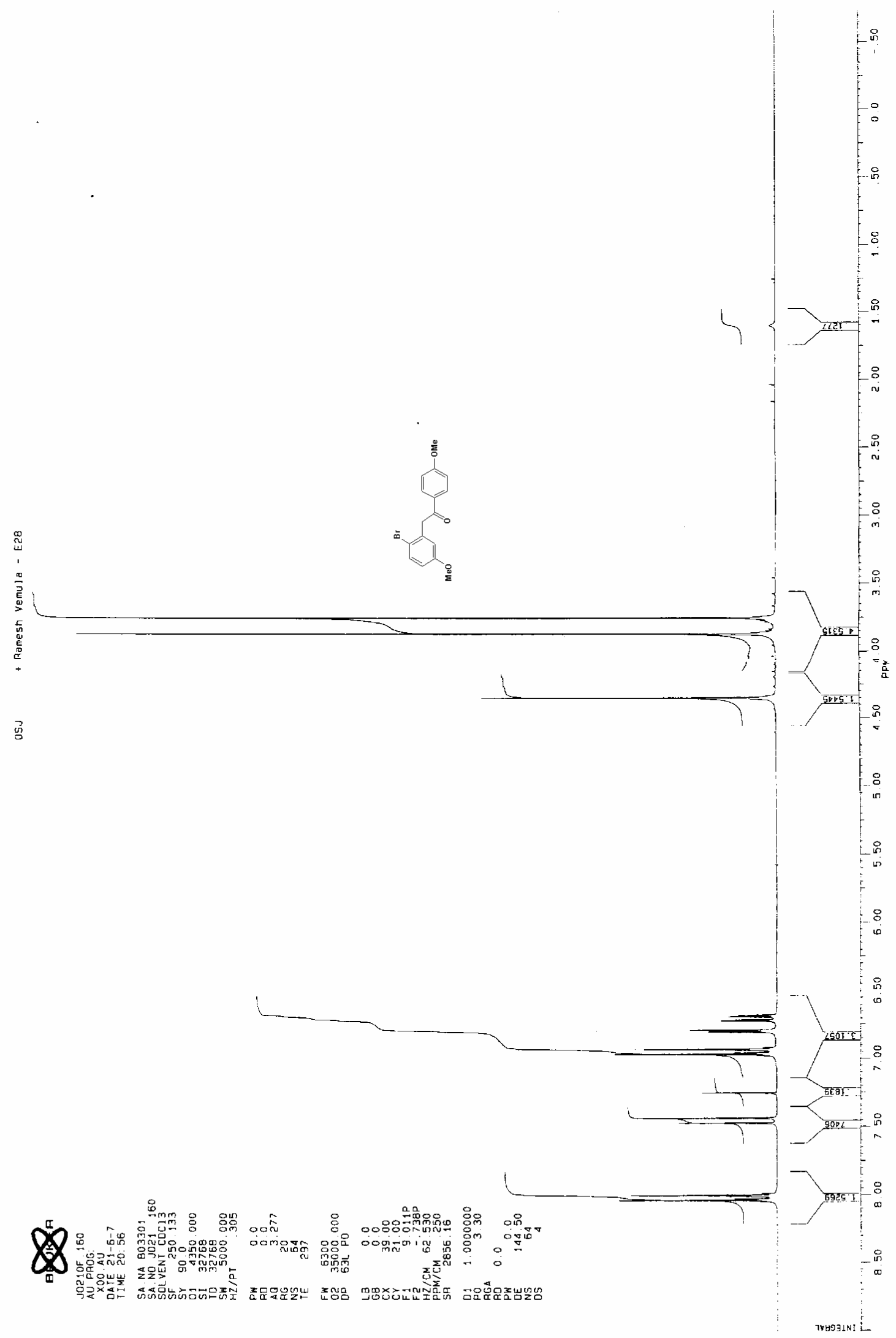


${ }^{1}$ H NMR Spectrum of compound 2

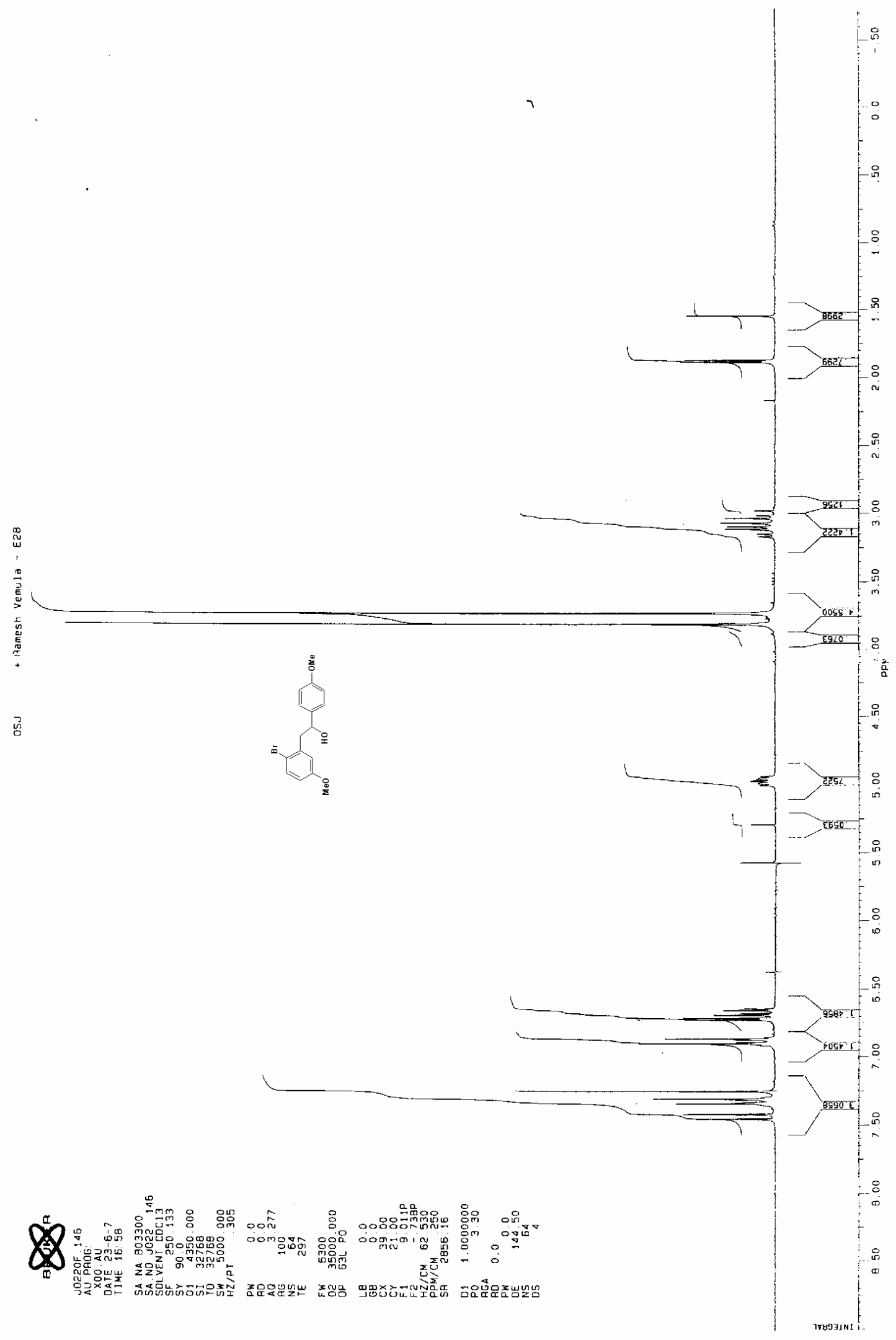


${ }^{1}$ H NMR Spectrum of compound 8
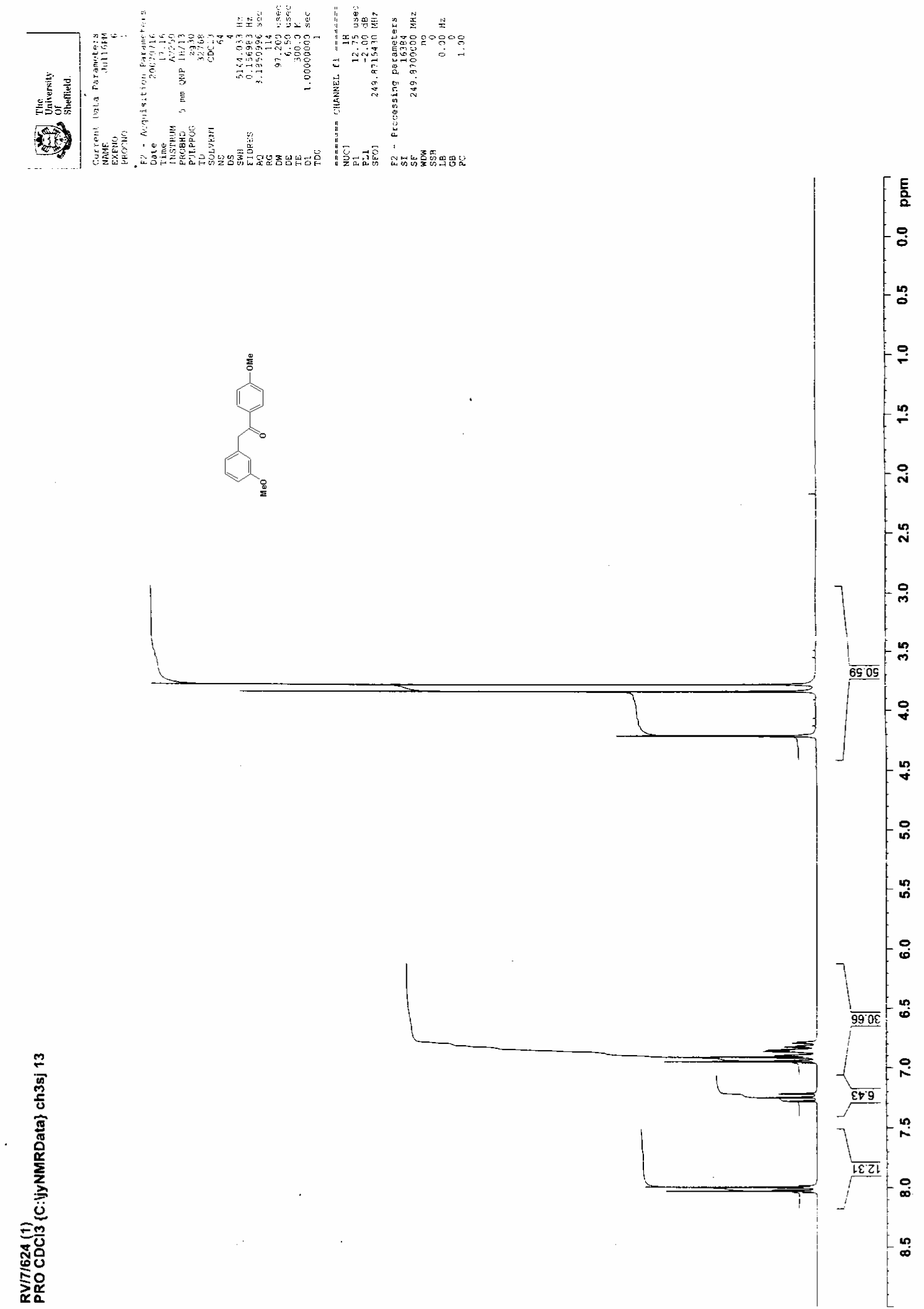
${ }^{1}$ H NMR Spectrum of compound 7
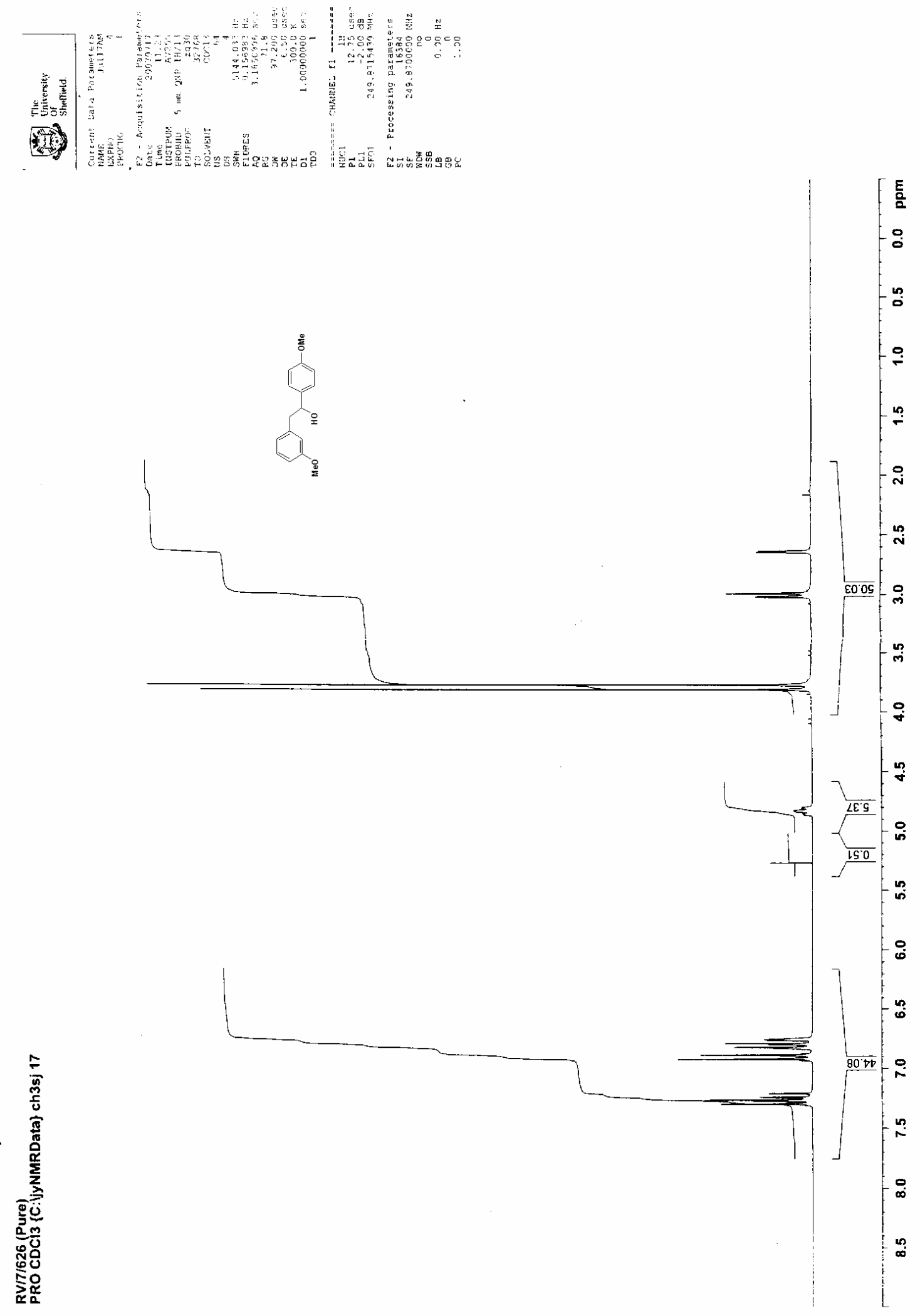
${ }^{1}$ H NMR Spectrum of compound 1
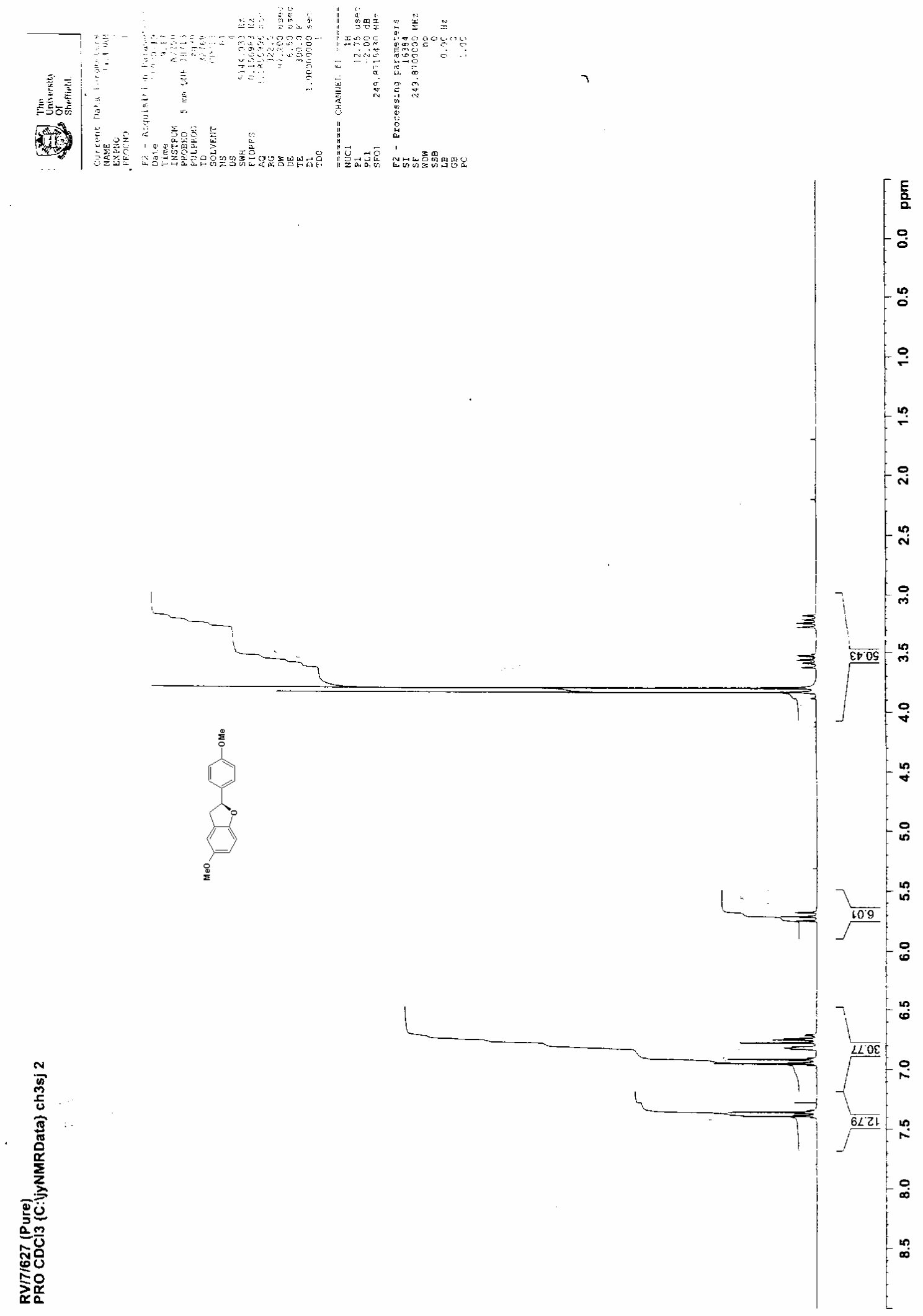


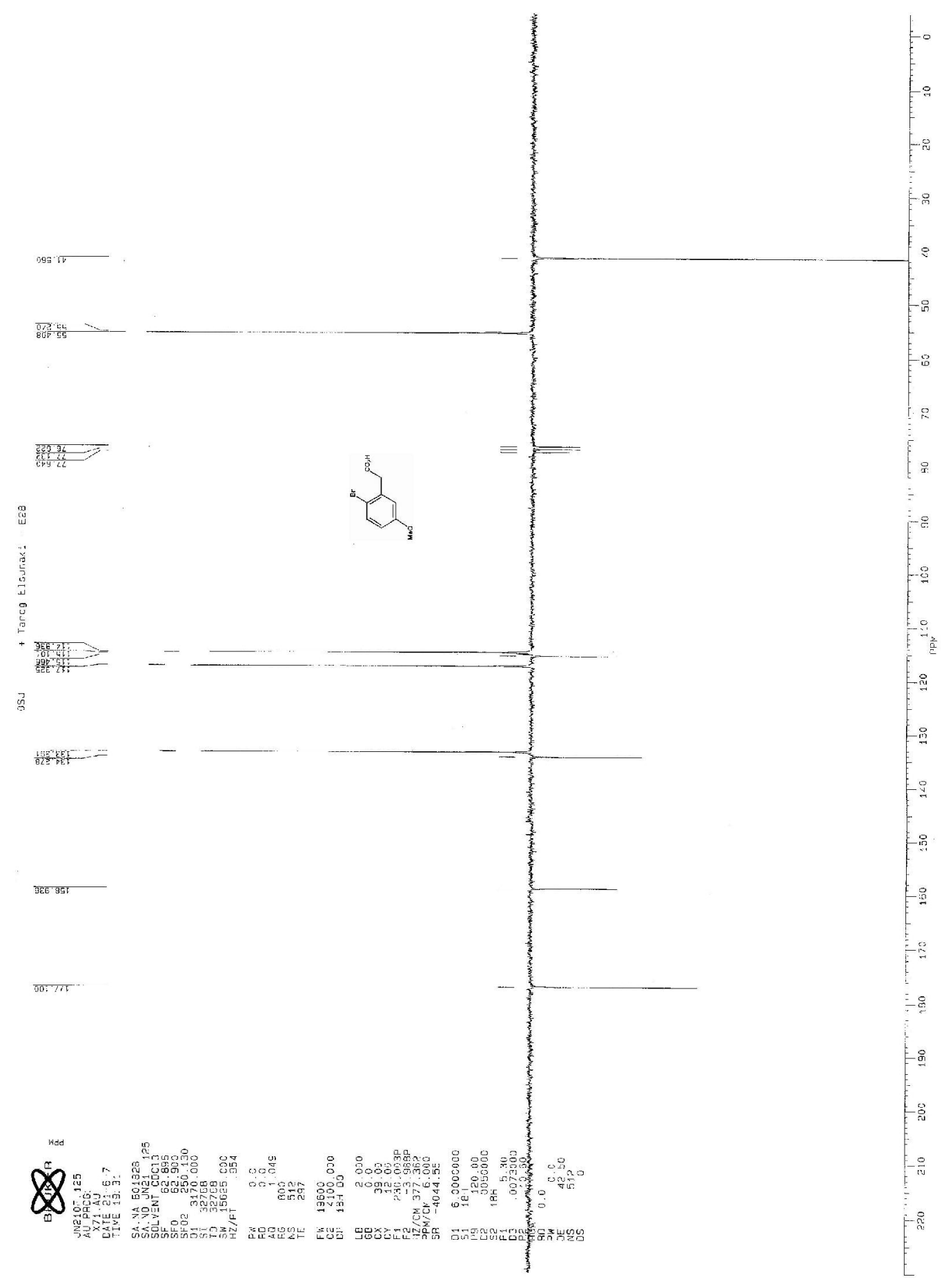


${ }^{13} \mathrm{C}$ NMR Spectrum of compound 3
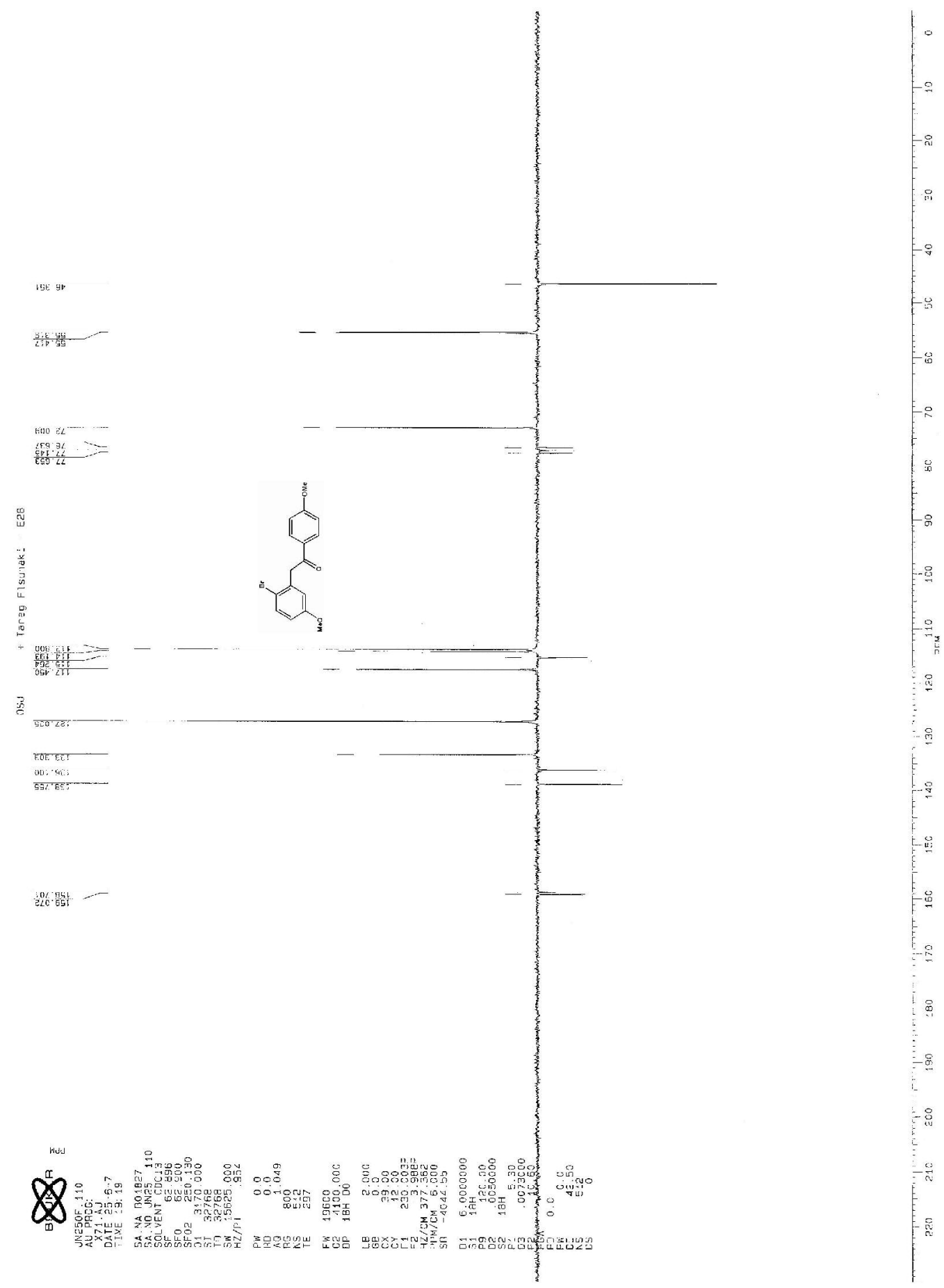
${ }^{13} \mathrm{C}$ NMR Spectrum of compound 2
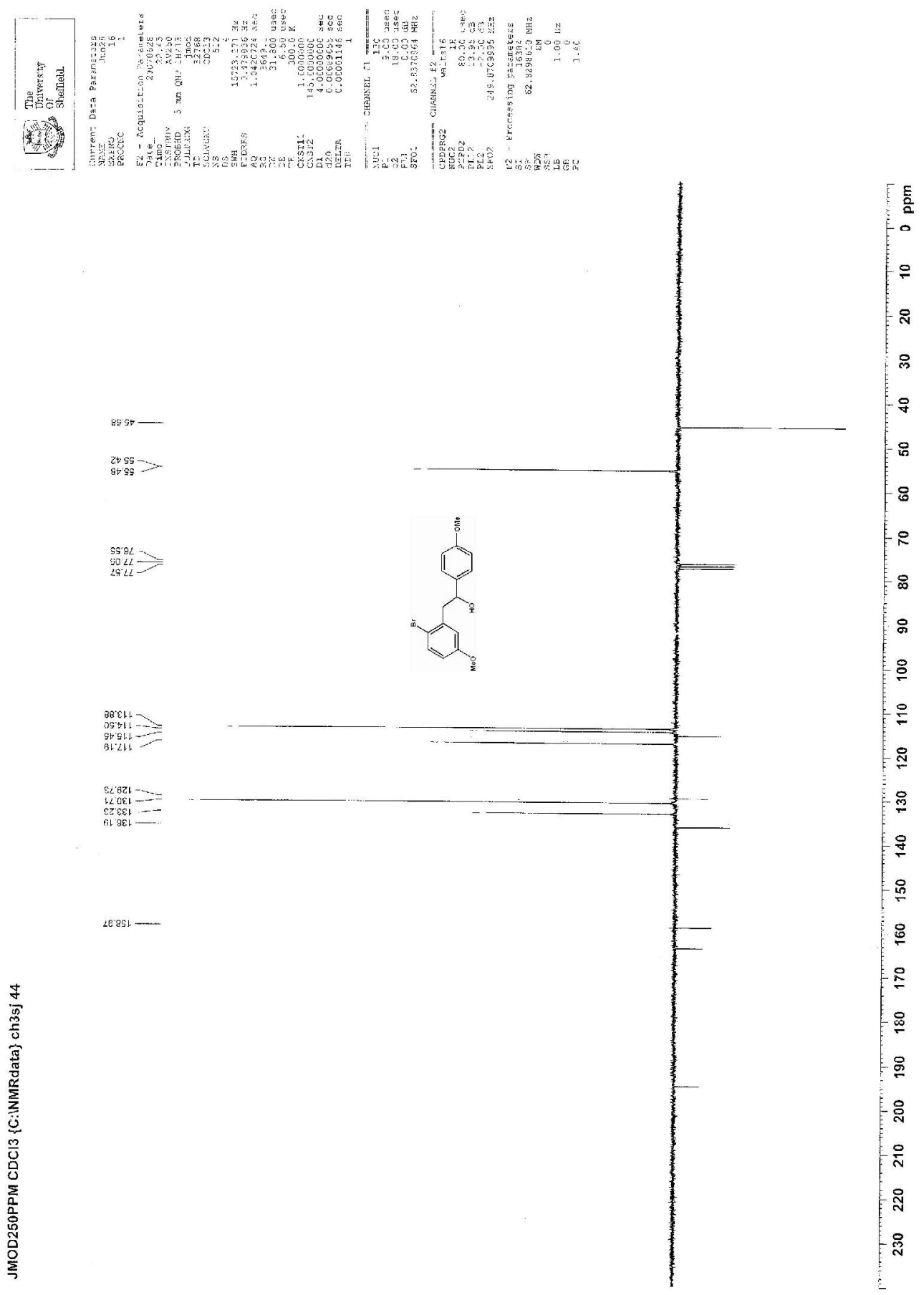
${ }^{13} \mathrm{C}$ NMR Spectrum of compound 8

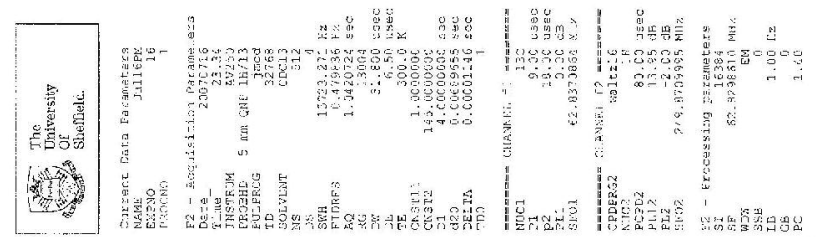

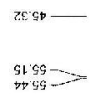
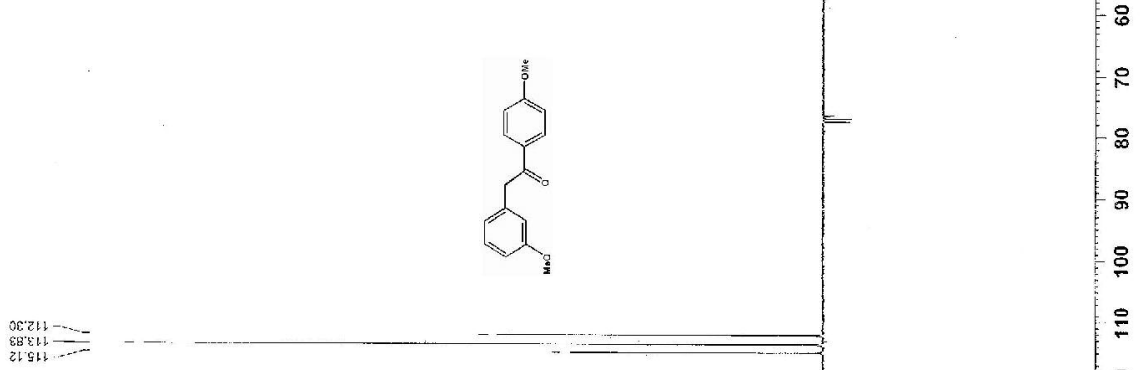

a닌 -

믄다-

95.9\&1 - -

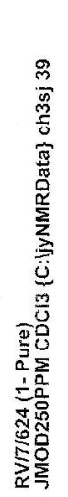

89:al-

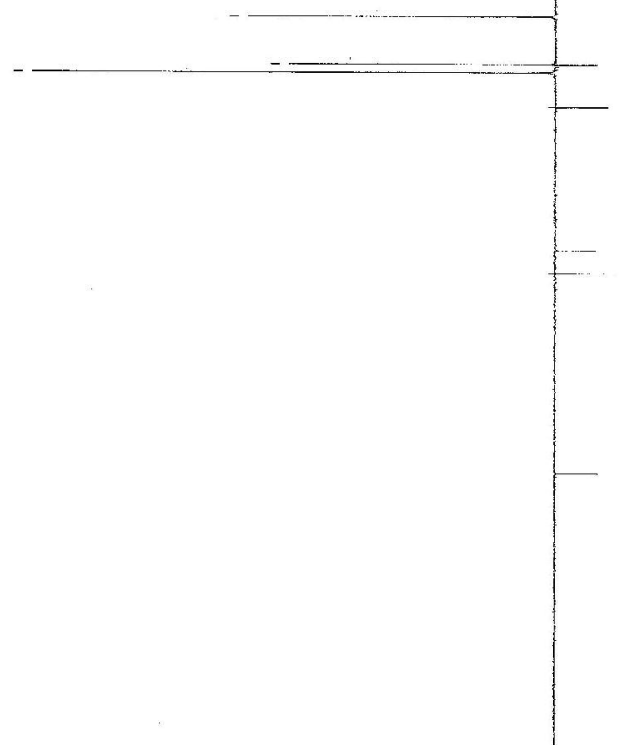


${ }^{13} \mathrm{C}$ NMR Spectrum of compound 7
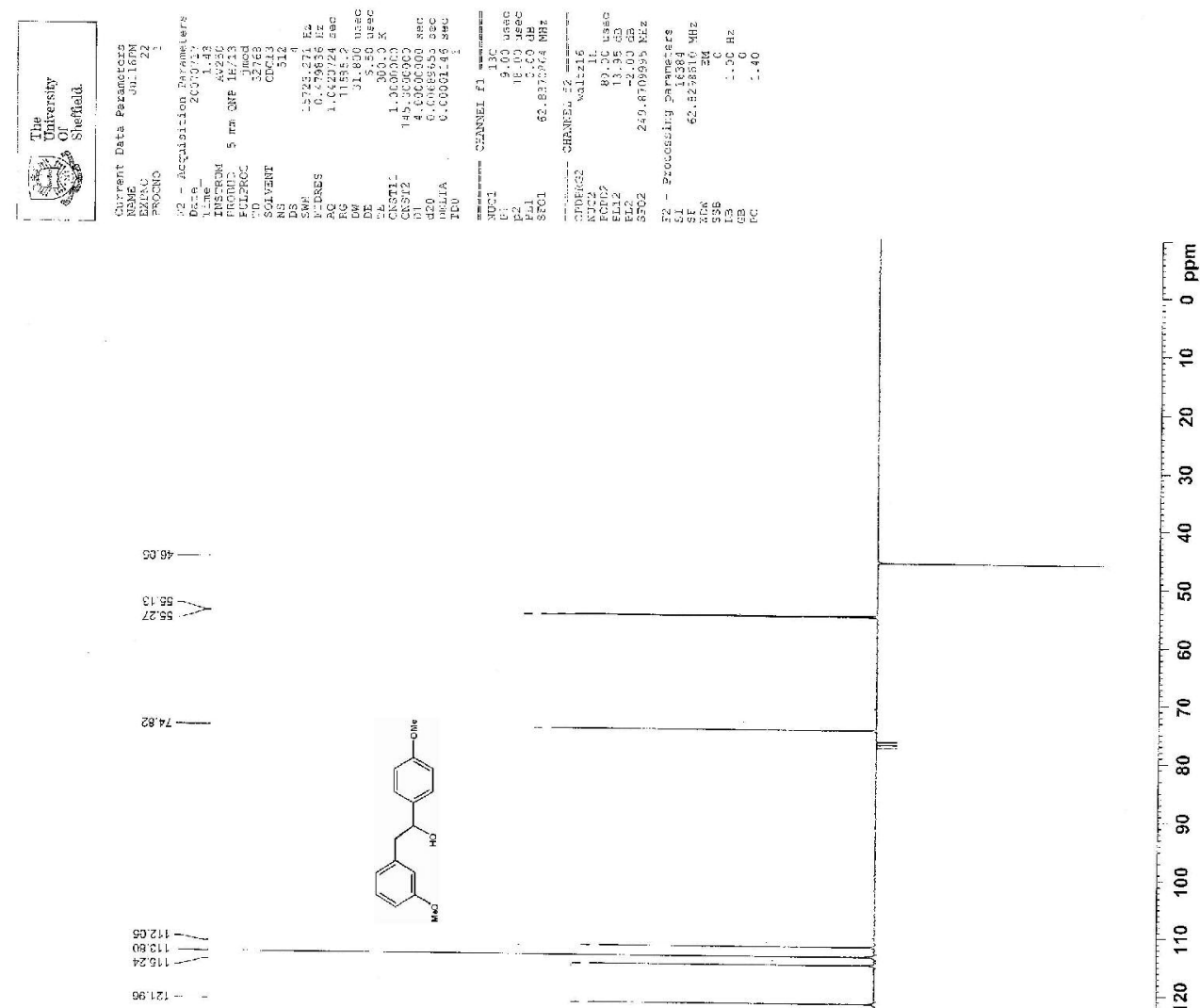

$$
=
$$

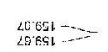

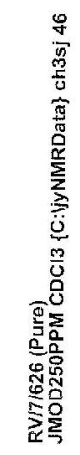


${ }^{13} \mathrm{C}$ NMR Spectrum of compound 1

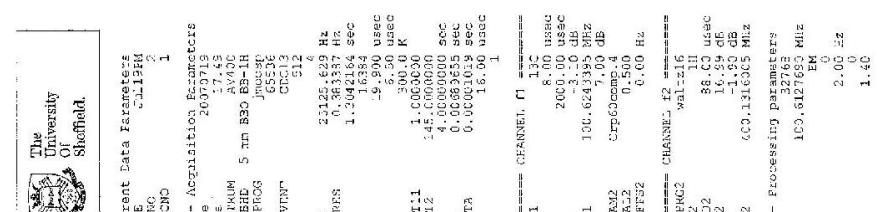

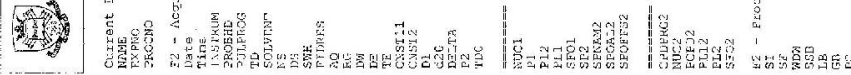

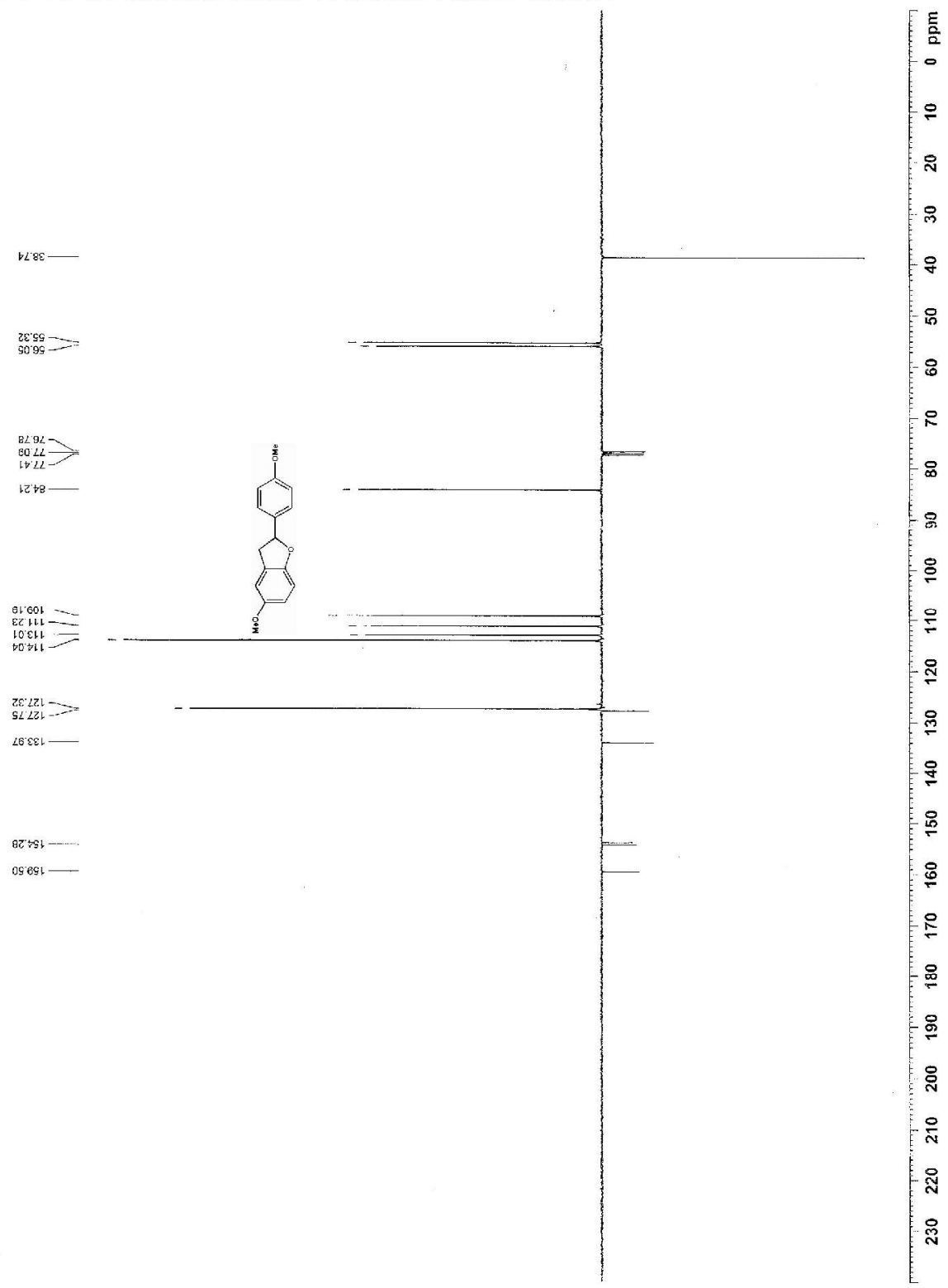




\section{HPLC Chromatogram of $(S)-2$ crude reaction mixture}

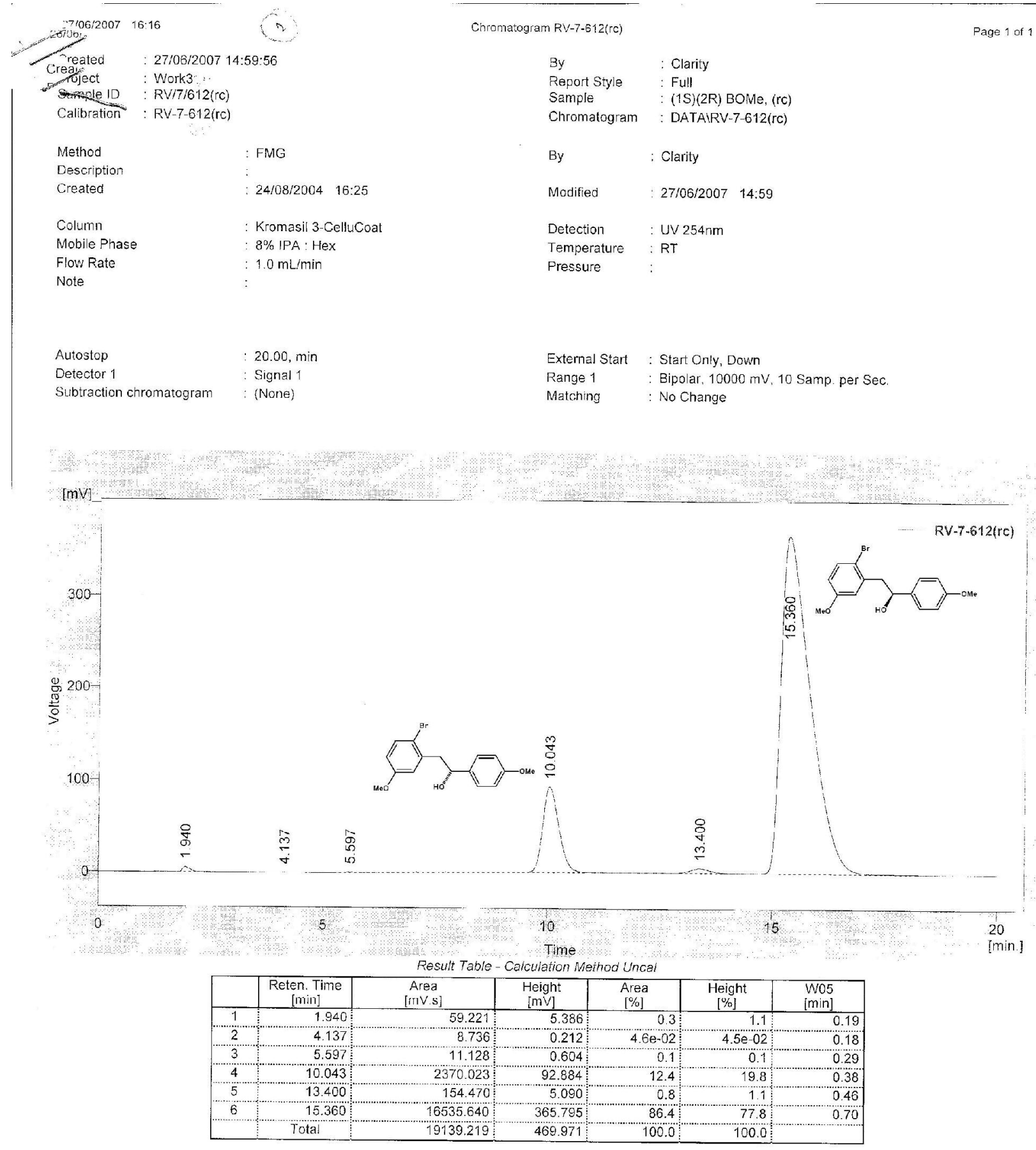




\section{HPLC Chromatogram of $(R)-2$ crude reaction mixture}

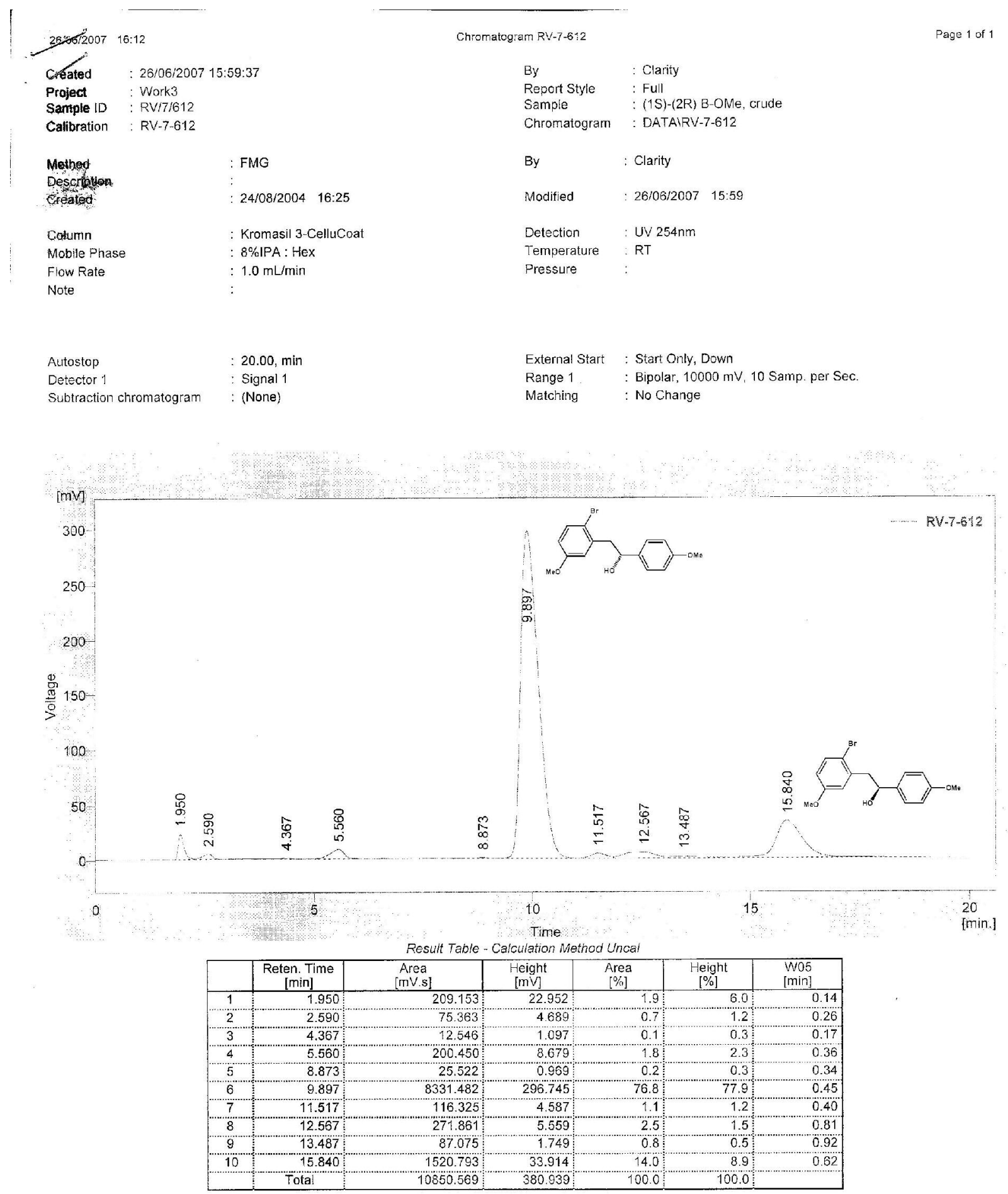




\section{GC Chromatogram of Synthetic (S)-1}

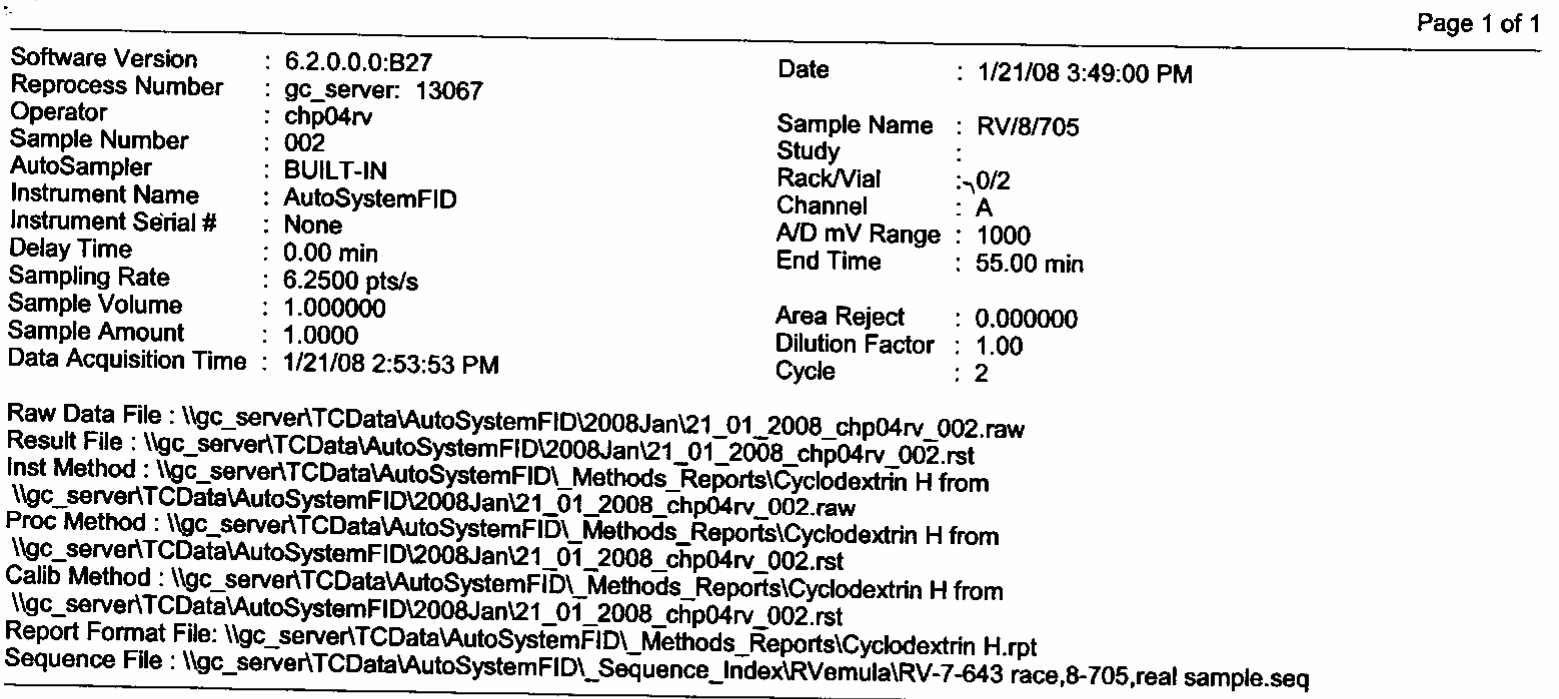

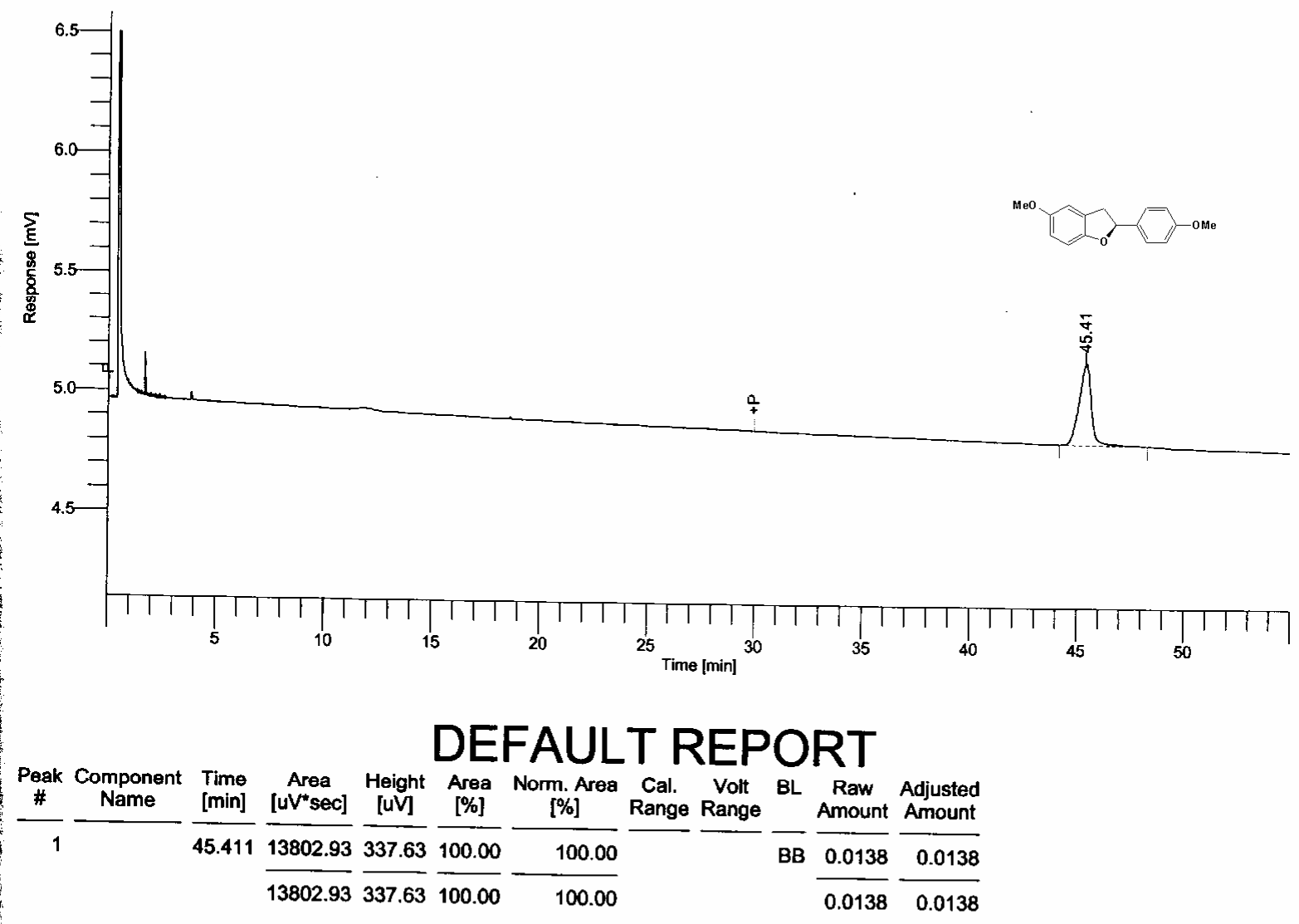




\section{GC Chromatogram of $(R)-1$}

Page 1 of 1

\begin{tabular}{ll}
\hline Software Version & $: 6.2 .0 .0 .0: B 27$ \\
Reprocess Number & $\vdots$ gc_server: 12544 \\
Operator & $:$ chp04rv \\
Sample Number & $: 003$ \\
AutoSampler & $\vdots$ BUILT-IN \\
Instrument Name & $:$ AutoSystemFID \\
Instrument Sèrial \# & $:$ None \\
Delay Time & $: 0.00 \mathrm{~min}$ \\
Sampling Rate & $: 6.2500 \mathrm{pts} / \mathrm{s}$ \\
Sample Volume & $: 1.000000$ \\
Sample Amount & $: 1.0000$ \\
Data Acquisition Time & $: 8 / 17 / 0712: 20: 08 \mathrm{PM}$
\end{tabular}

$\begin{array}{ll}\text { Date } & : \text { 8/17/07 1:13:15 PM } \\ \text { Sample Name } & : \text { RV/7637 } \\ \text { Study } & : 0 / 12 \\ \text { RackNial } & \text { A } \\ \text { Channel } & 1000 \\ \text { AD mV Range } & : 53.00 \mathrm{~min} \\ \text { End Time } & 5.000000 \\ \text { Area Reject } & : 0.00 \\ \text { Dilution Factor } & : 1.00 \\ \text { Cycle } & : 2\end{array}$

Raw Data File : Ilgc servertTCDatalAutoSystemFIDL2007Aug 117082007 chp04rv 003.raw

Result File : Ilgc_servertTCDataUutoSystemFIDL2007Augl17_08_2007_chp04rv_003.rst

Inst Method : Ilgc_serverITCDatalAutoSystemFIDI_Methods_ReportsiCyclodextrin $\mathrm{H}$ from

Ilgc_servertTCDatalAutoSystemFIDI2007Augl17 08_2007 chp04rv 003.raw

Proc Method : Igc_servertTCDatalAutoSystemFIDI_Methods_ReportsiCyclodextrin $\mathrm{H}$ from

ligc servenTCDatalAutoSystemFIDI2007Augl17 08 2007 chp04rv 003.rst

Calib Method: Ilgc_serverITCDataLAutoSystemFIDI Methods ReportsiCyclodextrin H from

Ilgc_serveriTCDataVutoSystemFIDL2007Augl17_08_2007_chp04n_003.rst

Report Format File: Ilgc_serverTCDatalAutoSystemFIDIMethods_ReportsiCyclodextrin H.rpt

Sequence File : Ilgc_servertTCDataUutoSystemFIDI_Sequence_IndexIRVemulalRV-7-643.seq

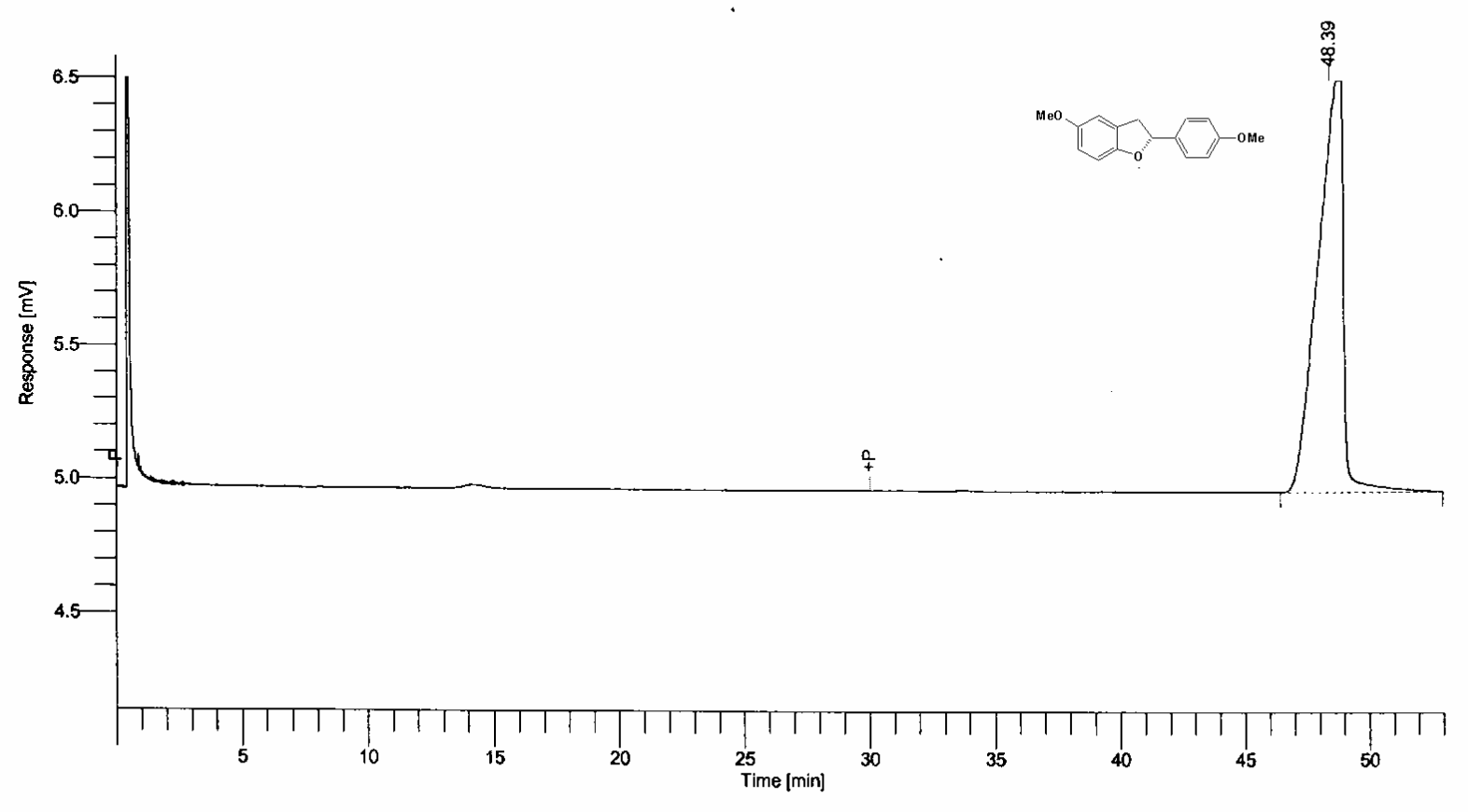

\section{DEFAULT REPORT}

\begin{tabular}{|c|c|c|c|c|c|c|c|c|c|c|c|}
\hline $\begin{array}{c}\text { Peak } \\
\#\end{array}$ & $\begin{array}{c}\text { Component } \\
\text { Name }\end{array}$ & $\begin{array}{l}\text { Time } \\
\text { [min] }\end{array}$ & $\begin{array}{c}\text { Area } \\
{\left[\mathrm{uV}^{*} \text { sec] }\right.}\end{array}$ & $\begin{array}{l}\text { Height } \\
\text { [uV] }\end{array}$ & $\begin{array}{c}\text { Area } \\
{[\%]}\end{array}$ & $\begin{array}{c}\text { Norm. Area } \\
{[\%]}\end{array}$ & $\begin{array}{c}\text { Cal. } \\
\text { Range }\end{array}$ & $\begin{array}{c}\text { Volt } \\
\text { Range }\end{array}$ & $\mathrm{BL}$ & $\begin{array}{l}\text { Row } \\
\text { Amount }\end{array}$ & $\begin{array}{l}\text { Adjusted } \\
\text { Amount }\end{array}$ \\
\hline \multirow[t]{2}{*}{1} & & 48.386 & 111854.09 & 1305.89 & 100.00 & 100.00 & & & BB & 0.1119 & 0.1119 \\
\hline & & & 111854.09 & 1305.89 & 100.00 & 100.00 & & & & 0.1119 & .7 \\
\hline
\end{tabular}




\section{GC Chromatogram of racemic corsifuran 1}

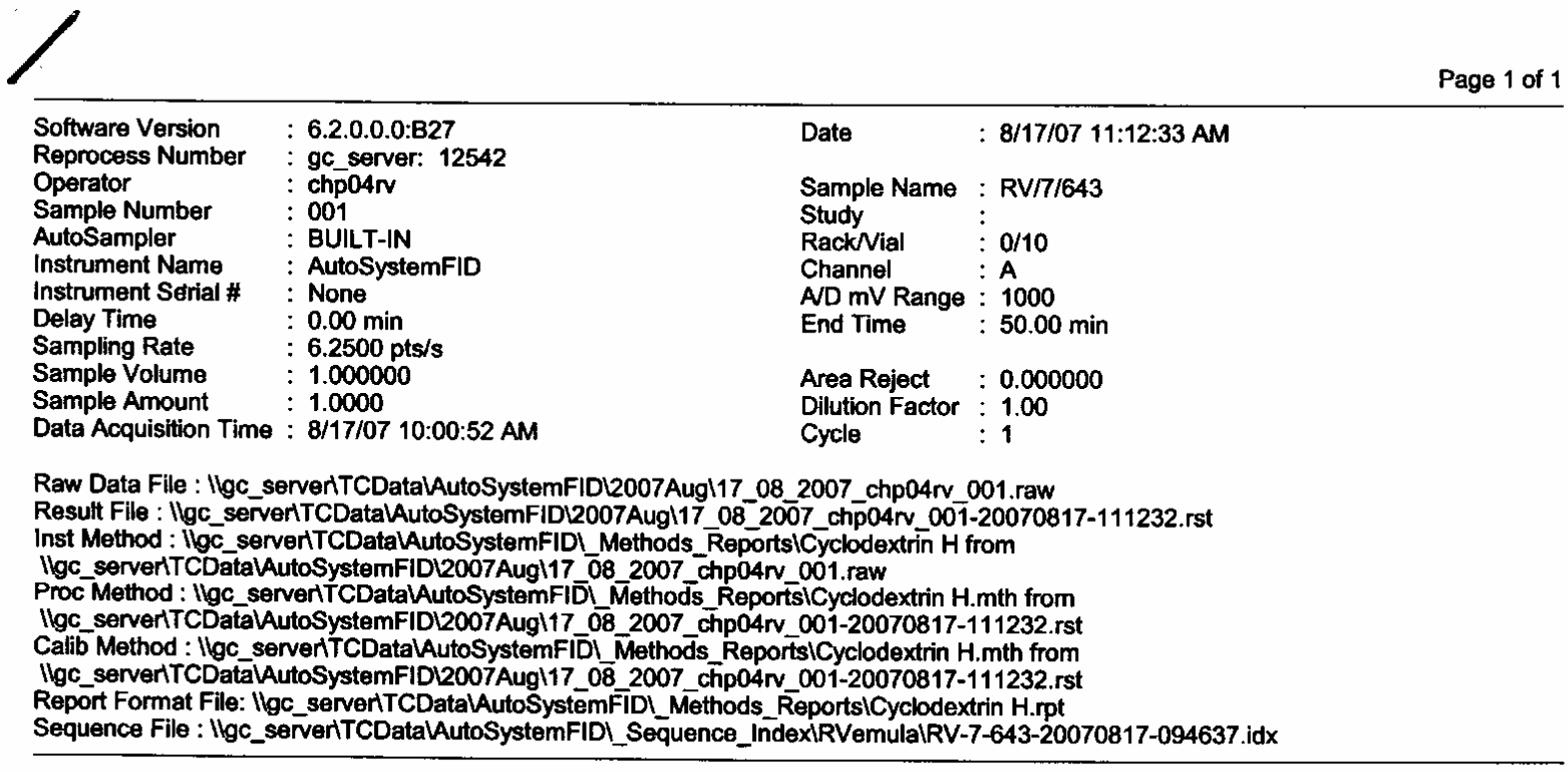

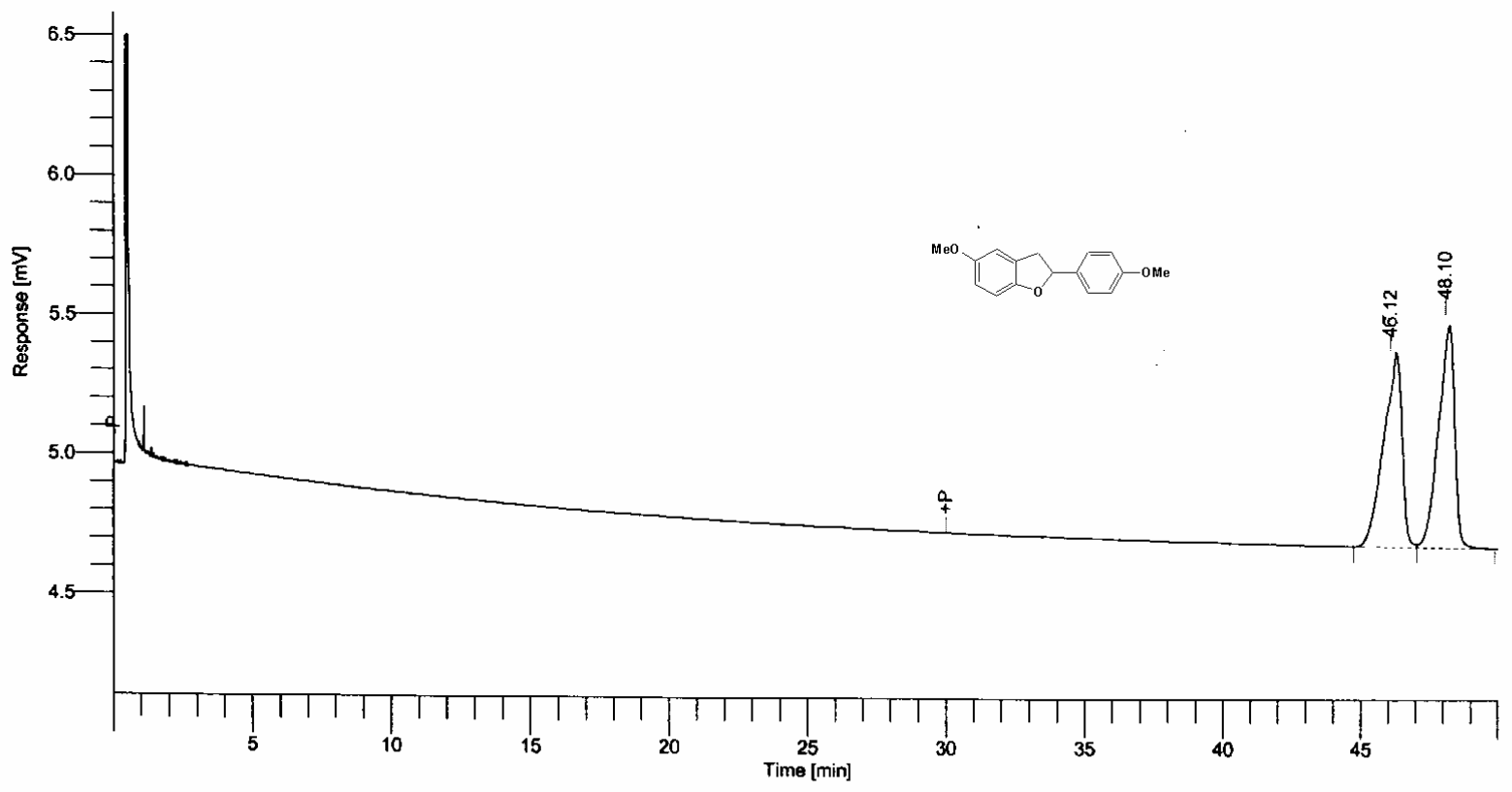

\section{DEFAULT REPORT}

\begin{tabular}{|c|c|c|c|c|c|c|c|c|c|c|c|}
\hline $\begin{array}{c}\text { Peak } \\
\#\end{array}$ & $\begin{array}{c}\text { Component } \\
\text { Name }\end{array}$ & $\begin{array}{l}\text { Time } \\
\text { [min] }\end{array}$ & $\begin{array}{c}\text { Area } \\
{\left[\mathrm{uV}^{*} \sec \right]}\end{array}$ & $\begin{array}{c}\text { Height } \\
\text { [uV] }\end{array}$ & $\begin{array}{l}\text { Area } \\
{[\%]}\end{array}$ & $\begin{array}{c}\text { Norm. Area } \\
{[\%]}\end{array}$ & $\begin{array}{c}\text { Cal. } \\
\text { Range }\end{array}$ & $\begin{array}{c}\text { Volt } \\
\text { Range }\end{array}$ & $\mathrm{BL}$ & $\begin{array}{l}\text { Raw } \\
\text { Amount }\end{array}$ & $\begin{array}{l}\text { Adjusted } \\
\text { Amount }\end{array}$ \\
\hline & & $\begin{array}{l}46.117 \\
48.103\end{array}$ & $\begin{array}{l}32352.73 \\
32966.69\end{array}$ & $\begin{array}{l}544.61 \\
684.56\end{array}$ & $\begin{array}{l}49.53 \\
50.47\end{array}$ & $\begin{array}{l}49.53 \\
50.47\end{array}$ & & & $\begin{array}{l}\text { BV } \\
\text { VB }\end{array}$ & $\begin{array}{l}0.0324 \\
0.0330\end{array}$ & $\begin{array}{l}0.0324 \\
0.0330\end{array}$ \\
\hline & & & 65319.42 & 1229.17 & 100.00 & 100.00 & & & & 0.0653 & 0.0653 \\
\hline
\end{tabular}




\section{GC Chromatogram of natural corsifuran 1}

\begin{tabular}{|c|c|c|c|}
\hline $\begin{array}{l}\text { Software Version } \\
\text { Reprocess Number } \\
\text { Operator } \\
\text { Sample Number } \\
\text { AutoSampler } \\
\text { Instrument Name } \\
\text { Instrument Serial \# } \\
\text { Delay Time } \\
\text { Sampling Rate } \\
\text { Sample Volume } \\
\text { Sample Amount } \\
\text { Data Acquisition Time }\end{array}$ & $\begin{array}{l}\text { 6.2.0.0.0:B27 } \\
\text { gc_server: } 13069 \\
\text { chp04rv } \\
003 \\
\text { BUILT-IN } \\
: \text { AutoSystemFID } \\
\text { None } \\
0.00 \mathrm{~min} \\
6.2500 \mathrm{pts} / \mathrm{s} \\
1.000000 \\
1.0000 \\
1 / 21 / 083: 54: 59 \mathrm{PM}\end{array}$ & $\begin{array}{l}\text { Date } \\
\text { Sample Name } \\
\text { Study } \\
\text { RackNial } \\
\text { Channel } \\
\text { AD mV Range } \\
\text { End Time } \\
\\
\text { Area Reject } \\
\text { Dilution Factor } \\
\text { Cycle }\end{array}$ & $\begin{array}{l}: \text { RV/8/Real sample } \\
\vdots \\
: 0 / 3 \\
\mathrm{~A}^{\mathrm{A}} \\
1000 \\
: 55.00 \mathrm{~min} \\
\\
0.000000 \\
1.00 \\
3\end{array}$ \\
\hline
\end{tabular}

Raw Data File : llgc_serverTCDatałAutoSystemFIDL2008Janl21_01_2008_chp04r_003.raw

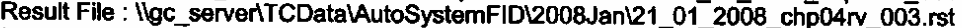
Inst Method : Ilgc_serveriTCDataVutoSystemFIDI Methods ReportsiCyclodextrin $\mathrm{H}$ from Ilgc_servertTCDatalAutoSystemFIDL2008Janl21_01_2008_chp04rv_003.raw

Proc Method : Ilgc_servertTCDatalAutoSystemFIDI_Methods_ReportsiCyclodextrin $\mathrm{H}$ from IIgC_serveriTCDatalAutoSystemFIDL2008Jan121_01_2008_chp04r_003.rst Calib Method : Ilgc_senverITCDatalAutoSystemFIDI Methods ReportsiCyclodextrin $\mathrm{H}$ from Ilgc_servertTCDatalAutoSystemFIDL2008Janl21_01_2008_chp04rv_003.rst

Report Format File: Ilgc_servertTCDatalAutoSystemFIDI_Methods_ReportsiCyclodextrin H.rpt Sequence File : Ilgc_serverITCDatałAutoSystemFIDI_Sequence_IndexIRVemulalRV-7-643 race,8-705,real sample.seq

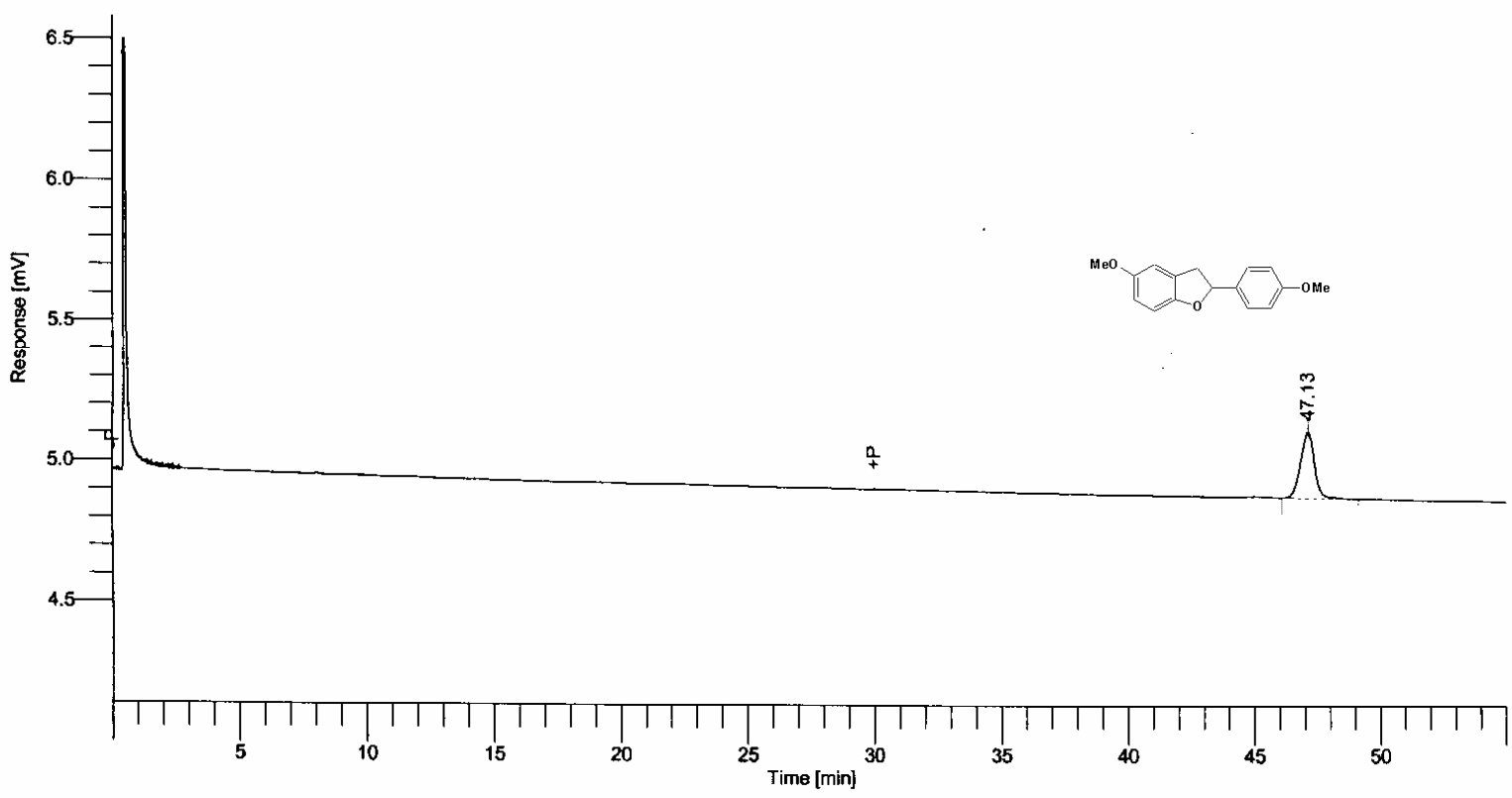

\section{DEFAULT REPORT}

\begin{tabular}{|c|c|c|c|c|c|c|c|c|c|c|c|}
\hline $\begin{array}{c}\text { Peak } \\
\#\end{array}$ & $\begin{array}{c}\text { Component } \\
\text { Name }\end{array}$ & $\begin{array}{l}\text { Time } \\
\text { [min] }\end{array}$ & $\begin{array}{c}\text { Area } \\
{\left[\mathrm{uV}^{*} \mathrm{sec}\right]}\end{array}$ & $\begin{array}{l}\text { Height } \\
\text { [uV] }\end{array}$ & $\begin{array}{c}\text { Area } \\
{[\%]}\end{array}$ & $\begin{array}{c}\text { Norm. Area } \\
{[\%]}\end{array}$ & $\begin{array}{c}\text { Cal. } \\
\text { Range }\end{array}$ & $\begin{array}{c}\text { Volt } \\
\text { Range }\end{array}$ & BL & $\begin{array}{c}\text { Raw } \\
\text { Amount }\end{array}$ & $\begin{array}{l}\text { Adjusted } \\
\text { Amount }\end{array}$ \\
\hline \multirow[t]{2}{*}{1} & & 47.132 & 9153.96 & 234.26 & 100.00 & 100.00 & & & BB & 0.0092 & 0.0092 \\
\hline & & & 9153.96 & 234.26 & 100.00 & 100.00 & & & & 0.0092 & 0.0092 \\
\hline
\end{tabular}




\section{X-Ray Structure of $(R)-1$}

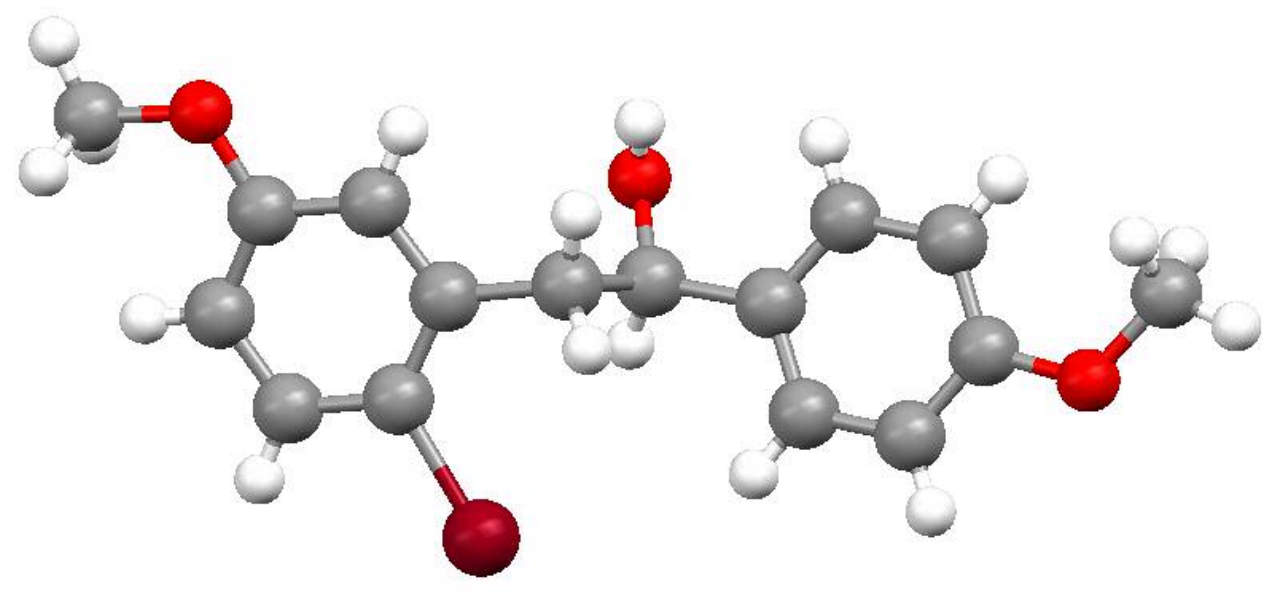

X-Ray Structure of $(S)-1$

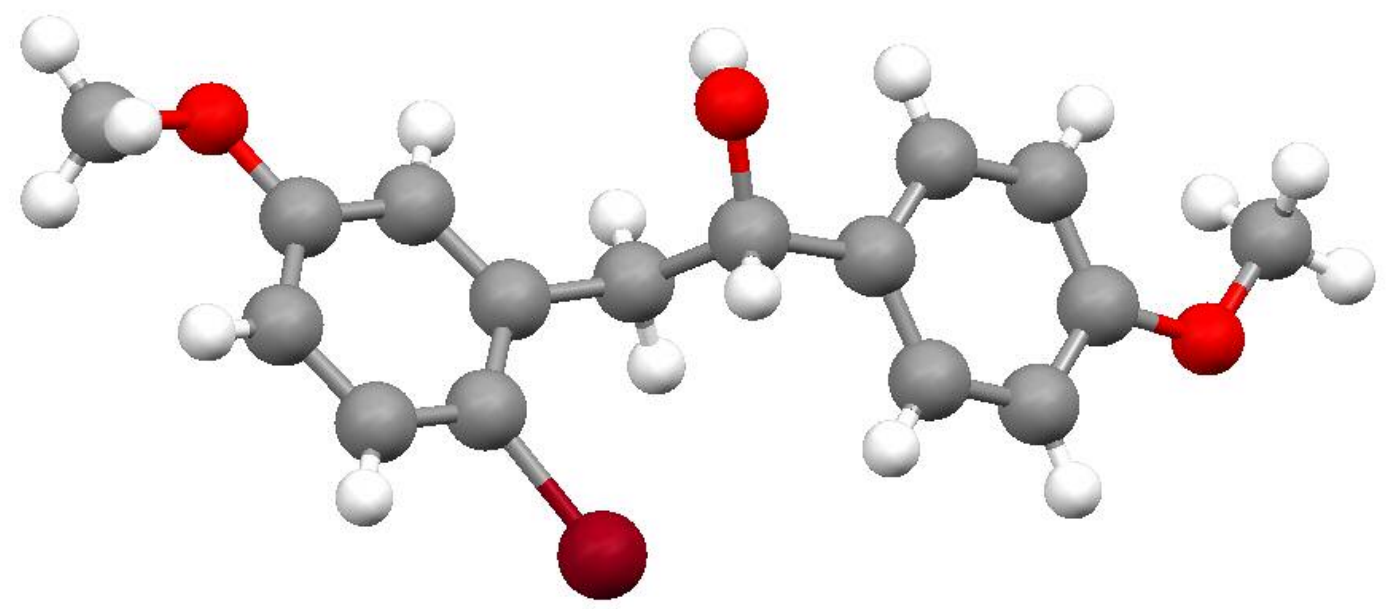

\section{References}

Lebegue, N.; Bethegnies, G.; Berthelot, P. Synth. Comm. 2004, 34, 1041-1048.

Percec, V.; Zuber, M. J. Polym. Sci., A: Polymer Chemistry 1992, 30, 997-

1016

3 Napolitano, E.; Ramacciotti, A.; Fiaschi, R. Gazz. Chem. Ital. 1988, 118, 101103.

4 Personal communication; von Reuß, S. H. Institute Für Organische Chemie, Universität Hamburg. Martin-Luther-King-Platz 6,D-29146 Hamburg, Germany 